\title{
Effective Field Theory for Dilute Fermions with Pairing
}

\author{
R.J. Furnstahl* \\ Department of Physics, The Ohio State University, Columbus, OH 43210 \\ H.-W. Hammert \\ Helmholtz-Institut für Strahlen- und Kernphysik (Theorie), \\ Universität Bonn, Nußallee 14-16, D-53115 Bonn, Germany \\ S.J. Pugliat \\ SBIG (UK) PLC, Berkeley Square House, \\ London W1J 6BR, United Kingdom
}

(Dated: August 13, 2021)

\begin{abstract}
Effective field theory (EFT) methods for a uniform system of fermions with short-range, natural interactions are extended to include pairing correlations, as part of a program to develop a systematic Kohn-Sham density functional theory (DFT) for medium and heavy nuclei. An effective action formalism for local composite operators leads to a free-energy functional that includes pairing by applying an inversion method order by order in the EFT expansion. A consistent renormalization scheme is demonstrated for the uniform system through next-to-leading order, which includes induced-interaction corrections to pairing.
\end{abstract}

PACS numbers: 24.10.Cn, 71.15.Mb, 21.60.-n, 31.15.-p

Keywords: Effective field theory, effective action, pairing

*Electronic address: furnstahl.1@osu.edu

${ }^{\dagger}$ Electronic address: hammer@itkp.uni-bonn.de

${ }^{\ddagger}$ Electronic address: spuglia@sbiguk.com 


\section{INTRODUCTION}

In previous work, effective field theory (EFT) [1, 2, 3, 4, 4, 5, 6] was applied to the normal ground state of a dilute Fermi system with short-range interactions [7, 8] and subsequently extended to finite systems as a realization of Kohn-Sham density functional theory (DFT) [9, 10, 11]. These studies are part of a program to go beyond mean-field approaches to finite many-body systems while maintaining their computational simplicity and phenomenological strengths. Our ultimate goal is to systematically describe medium and heavy nuclei using EFT and renormalization group methods, including nuclei far from stability where EFT power counting and error estimates are desirable to enable reliable extrapolations. For nuclei as well as trapped systems of atomic gases, attractive interactions cause the normal ground state to be unstable to the formation of Cooper pairs. In the present work, we consider an extension of the EFT formalism for uniform systems to allow for pairing correlations in a way that is also consistent with Kohn-Sham DFT.

Our strategy is to apply an effective action formalism [12, 13, 14] to calculate the free energy order by order in an EFT expansion. The effective action formalism is often used in condensed matter applications to discuss superconductivity, starting from a path integral with a four-fermion contact interaction (see, for example, [15, 16]). In the standard approach, a pairing field is introduced as a charged scalar auxiliary field and shifted to eliminate the four-fermion term, leaving only a Gaussian integral over the fermion fields. After performing this integral, a conventional effective action is derived by a functional Legendre transformation with respect to the auxiliary field. A minimum at a nonzero expectation value of this field (which is proportional to the pairing gap for a short-range interaction at leading order) indicates spontaneous symmetry breaking of the phase symmetry related to fermion number conservation, which implies the normal ground state is unstable to pairing and determines the "superconducting" ground state.

Instead of introducing an auxiliary pairing field, we couple an external source (or, more generally, a pair of complex conjugate sources) to the local, composite pair density [Eq. (19)]. In general this source would be space-time dependent, but for a uniform system in its ground state, which we consider here, the source can be treated as a constant. This external source is analogous to the source introduced for finite systems in Ref. [9], which was coupled to the composite fermion density. As in that case, we carry out a functional Legendre transformation order by order in the EFT expansion via the inversion method introduced by Fukuda et al. [17, 18] (see Ref. [19] for an application to superconductivity). If we keep the space dependence of the sources and the corresponding densities, we obtain a generalization of the EFT-based density functional theory (DFT) formalism of Ref. [9] (see also Refs. [20, $21,22,23,24,25])$. In the present work, the limitation to uniform systems means that the inversion method parallels the construction used long ago by Kohn, Luttinger, and Ward [26, 27] to show the relationship of zero-temperature diagrammatic calculations to ones using the finite-temperature Matsubara formalism in the zero-temperature limit.

In Refs. [28] and [29], density functional theory for superconductivity was proposed using a nonlocal source coupled to the nonlocal pair density. While this approach can be embedded in an effective action framework, we first want to explore the use of local sources, which are greatly preferred for finite nuclei from a computational point of view. In particular, we seek a consistent generalization of the Skyrme-Hartree-Fock-Bogoliubov approach [30].

Potential problems arise, however, because the use of local composite operators and zerorange interactions leads to new ultraviolet divergences even at the mean-field (Hartree-Fock) 
level when pairing is included. Divergences at this order are not encountered when coupling an external source to the fermion density $\psi^{\dagger} \psi$, but appear now because the composite operators $\psi \psi$ and $\psi^{\dagger} \psi^{\dagger}$ need additional renormalization [31]. The divergences at leading order are symptomatic of generic problems identified long ago by Banks and Raby [32] that arise with effective potentials of local composite operators. (Such divergences would be avoided by working with nonlocal sources as in Refs. [28, 29].) These problems inhibited for many years the use of effective actions of composite operators although more recently Verschelde et al. [33, 34, 35] and Miransky et al. [36, 37, 38] have revived their use for relativistic field theories. In this work, we show how to remove the new UV divergences through next-to-leading-order (NLO) by adding a term quadratic in the pairing source, using a renormalization prescription compatible with the inversion method and conventional treatments of pairing at leading order.

The BCS gap equation for contact interactions has conventionally been renormalized at leading order (LO) by using the equation for free-space scattering, which has the same divergence [see Eqs. (54) and (60) $)$. The four-fermion coupling is eliminated in favor of the scattering length, leaving finite, renormalized equations. This approach was applied in Refs. [39] and [40] to obtain analytic formulas for the pairing gap and ground state energy at LO. In Ref. [40], dimensional regularization with minimal subtraction (DR/MS) was used. DR/MS was also used to good advantage for the dilute Fermi calculations of the normal ground state in Ref. [7]. In extending the formalism of Ref. [7] to include pairing, we use a more general subtraction scheme (called "power divergence subtraction," or PDS), that lets us verify explicitly that the generating functional, effective action, gap equation, and observables are renormalized order by order in the inversion approach. We give renormalized expressions up to next-to-leading order (NLO), which contain the universal correction to the pre-factor of the gap at low density, first calculated in Ref. [41] (see also Ref. [42]).

The plan of the paper is as follows. In Sect. II, we briefly rederive the EFT for a dilute Fermi gas in the effective action formalism using the inversion method of Kohn, Luttinger, and Ward [26]. In Sect. [II, the approach is generalized to include pairing by introducing a source coupled to the pairing density and then carrying out the Legendre transformation to get an effective action as a functional of fermion and pair densities. The construction of the EFT effective action functional via the inversion method starts with the zeroth order functional. In Sect. IV, we carry out the leading order (LO) calculation in detail, reproducing standard results and extending the dimensional regularization results of Ref. [40]. In Sect. V] we extend the calculation to next-to-leading order (NLO), which introduces the so-called "induced interaction". Section VI is a summary and some additional details are included in the Appendices.

\section{INVERSION METHOD WITHOUT PAIRING}

\section{A. Lagrangian and Generating Functional}

In contrast to the zero-temperature, Minkowski-space EFT treatment of dilute systems in Ref. [7], we work in Euclidean space in the Matsubara (imaginary time) formalism at inverse temperature $\beta$. We use a four-vector notation $x \equiv\{\tau, \mathbf{x}\}$, with the $\tau$ dependence usually

implicit, or $\widetilde{k} \equiv\left\{\omega_{n}, \mathbf{k}\right\}$ in momentum space, where $\omega_{n}$ is a fermion Matsubara frequency. Throughout this work, however, we will consider the zero temperature $(\beta \rightarrow \infty)$ limit, for which the differences from Ref. [7] are minor (and the results equivalent after frequency 
integrals are performed).

The EFT Lagrangian for a nonrelativistic spin-1/2 fermion field with spin-independent interactions is the same as in Ref. [7], but converted to Euclidean form (which means $t \rightarrow-i \tau$ and an overall minus sign in defining $\mathcal{L}_{E}$ and the interaction Lagrangian $\left.\mathcal{L}_{E}^{\text {int }}\right)$ :

$$
\begin{aligned}
\mathcal{L}_{E}= & \psi^{\dagger}\left[\frac{\partial}{\partial \tau}-\frac{\vec{\nabla}^{2}}{2 M}\right] \psi+\frac{C_{0}}{2}\left(\psi^{\dagger} \psi\right)^{2}-\frac{C_{2}}{16}\left[(\psi \psi)^{\dagger}\left(\psi \overleftrightarrow{\nabla}^{2} \psi\right)+\text { h.c. }\right] \\
& -\frac{C_{2}^{\prime}}{8}(\psi \stackrel{\leftrightarrow}{\nabla} \psi)^{\dagger} \cdot(\psi \stackrel{\leftrightarrow}{\nabla} \psi)+\ldots \\
\equiv & \psi^{\dagger}\left[\frac{\partial}{\partial \tau}-\frac{\vec{\nabla}^{2}}{2 M}\right] \psi+\mathcal{L}_{E}^{\operatorname{int}}\left(\psi^{\dagger}, \psi\right),
\end{aligned}
$$

where $\stackrel{\leftrightarrow}{\nabla}=\overleftarrow{\nabla}-\vec{\nabla}$ is the Galilean invariant derivative and h.c. denotes the Hermitian conjugate. ${ }^{1}$ The terms proportional to $C_{2}$ and $C_{2}^{\prime}$ contribute to $s$-wave and $p$-wave scattering, respectively, while the dots represent terms with more derivatives and/or more fields, as well as renormalization counterterms. The Lagrangian Eq. (1) is a particular canonical form, which can be reached via field redefinitions. For example, higher-order terms with time derivatives are omitted, as they can be eliminated in favor of terms with spatial derivatives (see, for example, Ref. [43]). We will restrict ourselves here to spin-1/2 (i.e., degeneracy $\nu=2$ ) and spin-independent interactions, which is sufficient to illustrate the formalism and the renormalization issues; generalizing to isospin and spin-dependent interations is straightforward. We also consider only the $C_{0}$ vertex here, but comment at the end on the inclusion of vertices with derivatives.

We first consider a Euclidean generating functional with chemical potential $\mu$ and external sources $\eta(x)$ and $\eta^{\dagger}(x)[16,44]$ :

$$
Z\left[\eta, \eta^{\dagger} ; \mu\right] \equiv e^{-W\left[\eta, \eta^{\dagger} ; \mu\right]}=\int D \psi_{\alpha} D \psi_{\alpha}^{\dagger} e^{-\int d^{4} x\left[\mathcal{L}_{E}-\mu \psi_{\alpha}^{\dagger}(x) \psi_{\alpha}(x)+\eta_{\alpha}^{\dagger}(x) \psi_{\alpha}(x)+\psi_{\alpha}^{\dagger}(x) \eta_{\alpha}(x)\right]},
$$

where $\int d^{4} x$ includes a $d \tau$ integration that runs from $-\beta / 2$ to $\beta / 2$ (to facilitate the $\beta \rightarrow \infty$ limit) and anti-periodic boundary conditions are imposed. For simplicity, normalization factors are considered to be implicit in the functional integration measure. (See Refs. [17, 18, 44 for more detailed treatments of similar path integrals.) We have written the spin index $\alpha$ explicitly in Eq. (2); we will interchangeably use $\alpha=\{1,2\}$ and $\alpha=\{\uparrow, \downarrow\}$ in the sequel to denote individual spins, and the spin indices will be left implicit where there is no chance of confusion. We have kept the chemical potential $\mu$ separate from the Lagrangian in Eq. (2) to emphasize its role as an external source. In the next section, we will add a corresponding source coupled to the pair density.

A conventional perturbative expansion is realized by removing the interaction terms from the path integral in (11) in favor of functional derivatives with respect to $\eta$ and $\eta^{\dagger}$ and performing the remaining Gaussian integration over $\psi$ and $\psi^{\dagger}$ [16, 44]:

$$
Z\left[\eta, \eta^{\dagger} ; \mu\right]=Z_{0} e^{-\int d^{4} x \mathcal{L}_{E}^{\mathrm{int}}\left[\delta / \delta \eta(x),-\delta / \delta \eta^{\dagger}(x)\right]} e^{\int d^{4} y d^{4} y^{\prime} \eta^{\dagger}(y) \mathcal{G}_{0}\left(y, y^{\prime}\right) \eta\left(y^{\prime}\right)}
$$

where the spin indices are implicit and we have introduced the noninteracting partition function $Z_{0}$. Explicit expressions for the Matusbara Green's function $\mathcal{G}_{0}$ in coordinate and

\footnotetext{
${ }^{1}$ We use units with $\hbar=1$.
} 

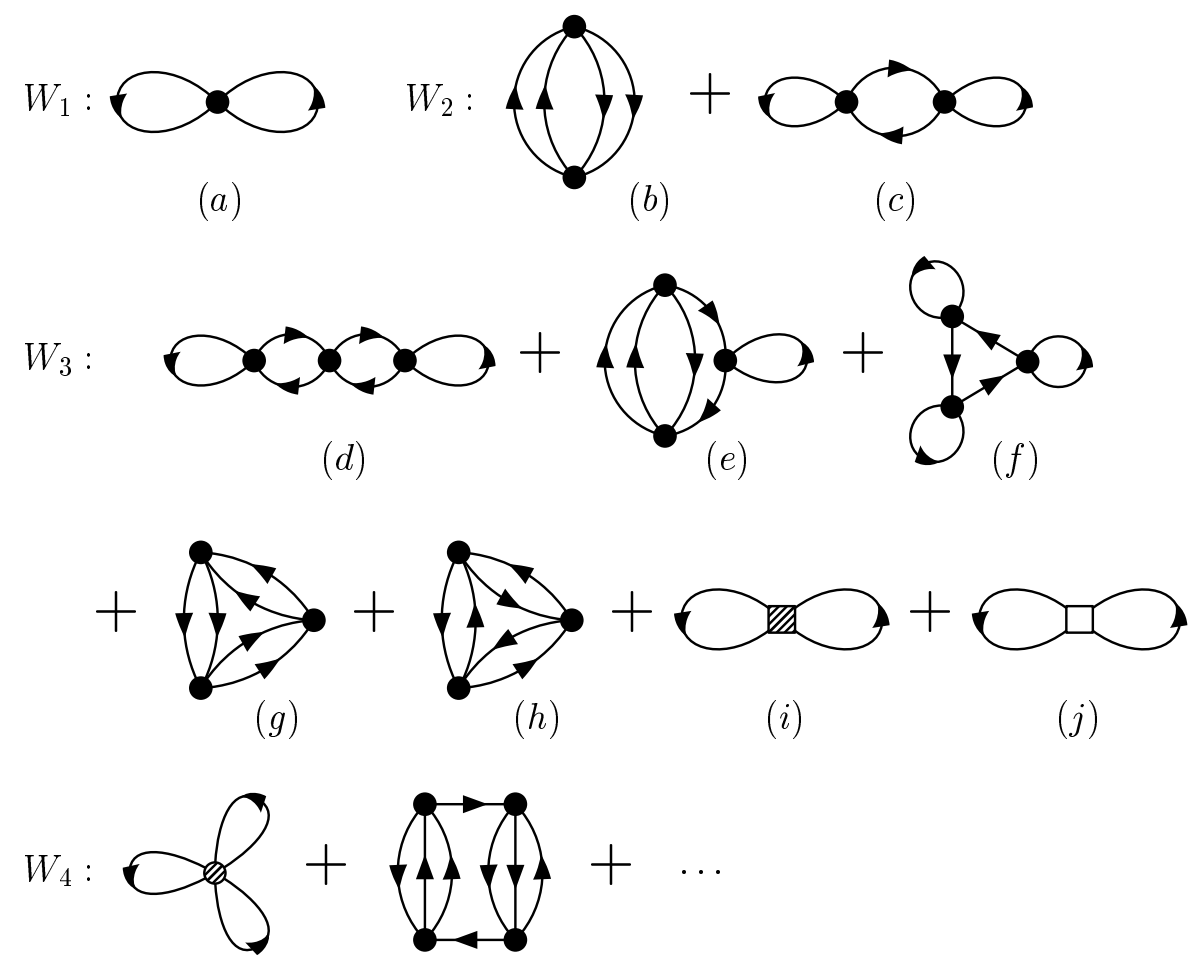

$(k)$

$(l)$

FIG. 1: Hugenholtz diagrams for the unrenormalized $W_{i}$ functionals in a homogeneous, dilute Fermi system. The vertices are defined in Fig. 5 ,

momentum space can be found in Ref. [44]. The linked-cluster theorem [44] shows that the difference of the interacting and noninteracting thermodynamic potentials $\Omega$ and $\Omega_{0}$ is given by the sum of connected diagrams from the expansion of $Z$, with the external sources $\eta^{\dagger}$ and $\eta$ set to zero at the end:

$$
\Omega(\mu, T, V)-\Omega_{0}(\mu, T, V)=\frac{1}{\beta}\left(W[0,0 ; \mu]-W_{0}[0,0 ; \mu]\right) .
$$

The connected Feynman diagrams that sum (with appropriate symmetry factors) to $W-W_{0}$, which are labled $W_{i}$ with $i \geq 1$, are shown in Fig. 1, with the fermion lines representing Matsubara propagators $\mathcal{G}_{0}$ with chemical potential $\mu$ [44]. The subscript $i$ labels the leading order to which $W_{i}$ contributes in the dilute EFT expansion. (In the DR/MS scheme, each diagram contributes to only one order but this is no longer true in DR/PDS.) The chemical potential can be determined for given $N$ by inverting the thermodynamic expression for the particle number, $N=\partial \Omega / \partial \mu$. The regularization and renormalization of divergences arising in the evaluation of the $W_{i}$ are described below.

\section{B. Kohn-Luttinger-Ward Inversion}

The Kohn-Luttinger-Ward (KLW) theorem [26, 27] relates the perturbative calculation of diagrams using the finite-temperature Matsubara formalism in the zero-temperature limit to 
the calculation of diagrams using zero-temperature perturbation theory, which was applied to the dilute Fermi gas in Ref. [7]. In particular, the diagrammatic expansion of $\Omega(\mu, V, T \rightarrow$ $0)$ is, under specified conditions, the same as the free-energy $F(N, V, T \equiv 0)$ evaluated using zero-temperature propagators with the non-interacting chemical potential $\mu_{0}$ and with anomalous diagrams omitted (see Ref. [7] for Feynman rules). The anomalous diagrams are those such as Fig. 1(c) and (d), which vanish in zero-temperature perturbation theory because they have particles and holes in the same intermediate single-particle state (i.e., factors of $\theta\left(\mu_{0}-\varepsilon_{\alpha}\right) \theta\left(\varepsilon_{\alpha}-\mu_{0}\right)=0$ appear $)$. The conditions of the theorem require that the symmetry of the non-interacting and interacting systems agree [44, 45].

A demonstration of the KLW theorem using an inversion method for the case of an electron gas is presented in Ref. [45]. We adapt this demonstration to the case of an EFT with a well-defined expansion. While we illustrate the procedure with the expansion relevant to a natural short-distance interaction (which is an expansion in the Fermi momentum $k_{\mathrm{F}}$ times the effective range parameters $a_{s}, r_{s}$, and so on [7]), the discussion is more general, requiring only a hierarchical expansion. Thus, for example, a nonperturbative (in diagrams) $1 / N$ expansion works just as well.

The basic plan is to carry out the conventional thermodynamic Legendre transformation:

$$
F(N)=\Omega(\mu)+\mu N
$$

with $\mu(N)$ obtained by inverting $N(\mu)=-(\partial \Omega / \partial \mu)_{T V}$, which determines the mean-number $N$ of particles. We expand each of the quantities in Eq. (5) about the non-interacting system:

$$
\begin{aligned}
\Omega(\mu) & =\Omega_{0}(\mu)+\Omega_{1}(\mu)+\Omega_{2}(\mu)+\cdots, \\
\mu & =\mu_{0}+\mu_{1}+\mu_{2}+\cdots, \\
F(N) & =F_{0}(N)+F_{1}(N)+F_{2}(N)+\cdots,
\end{aligned}
$$

where the subscript indicates the order of the expansion. (Note that the subscript is just a counting parameter that does not have to correspond to a power series in the expansion parameter; e.g., in the Coulomb case the expansion parameter is $e^{2}$ but $\Omega_{2}$ has both an $e^{4}$ term and the correlation energy of order $e^{4} \ln e$.) The non-interacting system refers to a system of zeroth order in the EFT expansion parameter. This means the zeroth-order system has no internal interactions among the particles, but it can include external sources (we exploit this freedom below). Each $\Omega_{i}(\mu)$ is given by $(1 / \beta) W_{i}[0,0 ; \mu]$, where the $W_{i}$ 's correspond to the diagrams in Fig. 1.

The number of particles is

$$
N(\mu, T, V)=-\left(\frac{\partial \Omega}{\partial \mu}\right)_{T V}=-\frac{\partial \Omega_{0}}{\partial \mu}-\frac{\partial \Omega_{1}}{\partial \mu}-\frac{\partial \Omega_{2}}{\partial \mu}+\cdots
$$

Note that we could simply use the unexpanded first equality in Eq. (9) together with the series in Eq. (6) , since they define a parametric relation between $N$ and $\Omega$ in terms of $\mu$ [45]. Since we have in mind the extension to finite systems, we pursue an alternative that generalizes to Kohn-Sham density functional theory (DFT). To carry this out, we perform the inversion in Eq. (9) order by order in the EFT expansion, treating $N$ as order zero in the expansion. (That is, we ensure there are no corrections to $N$ at higher order.) This means that the zeroth order equation,

$$
N=-\left[\frac{\partial \Omega_{0}}{\partial \mu}\right]_{\mu=\mu_{0}}
$$


is the only equation to which $N$ contributes (by construction). Thus the "exact" $N$ is obtained from the non-interacting thermodynamic potential. This might not sound impressive, but the analogous situation holds when we generalize $\mu$ to be position dependent or coupled to the pair density in a finite system. In these cases, it is the exact, spatially dependent fermion or pair density (with appropriate renormalization conditions, see below) that is obtained from a non-interacting system with a single-particle potential that is the generalization of $\mu_{0}$. This is precisely the description of the Kohn-Sham system (see Refs. [9] and [10] for details on carrying out the DFT case without pairing).

Equation (10) determines $N\left(\mu_{0}\right)$ at any temperature, from which we can find $\mu_{0}(N)$ for any system for which we can identify $\Omega_{0}$ (the inversion is unique since $\mu_{0}$ is a monotonic function of $N$ [45]). If we have a uniform system with no external sources, $\mu_{0}$ is the chemical potential of a noninteracting Fermi gas at temperature $T$ with density $N / V$. In particular, at $T=0$ with no external potential and spin-isospin degeneracy $\nu$,

$$
\mu_{0}(N)=\left(6 \pi^{2} N / \nu V\right)^{2 / 3} \equiv k_{\mathrm{F}}^{2} / 2 M \equiv \varepsilon_{\mathrm{F}}^{0} .
$$

The first-order equation extracted from Eq. (9) has two terms, which lets us solve for $\mu_{1}$ in terms of known (from diagrams) functions of $\mu_{0}$ :

$$
0=\left[\frac{\partial \Omega_{1}}{\partial \mu}\right]_{\mu=\mu_{0}}+\mu_{1}\left[\frac{\partial^{2} \Omega_{0}}{\partial \mu^{2}}\right]_{\mu=\mu_{0}} \Longrightarrow \mu_{1}=-\frac{\left[\partial \Omega_{1} / \partial \mu\right]_{\mu=\mu_{0}}}{\left[\partial^{2} \Omega_{0} / \partial \mu^{2}\right]_{\mu=\mu_{0}}} .
$$

At second order, we can isolate and solve for $\mu_{2}$, eliminating $\mu_{1}$ using (12). This pattern continues to all orders: $\mu_{i}$ is determined by functions of $\mu_{0}$ only.

Now we apply the inversion to $F=\Omega+\mu N$ :

$$
\begin{aligned}
F(N)= & \underbrace{\Omega_{0}\left(\mu_{0}\right)+\mu_{0} N}_{F_{0}}+\underbrace{\mu_{1}\left[\frac{\partial \Omega_{0}}{\partial \mu}\right]_{\mu=\mu_{0}}+\Omega_{1}\left(\mu_{0}\right)+\mu_{1} N}_{F_{1}} \\
& +\underbrace{\mu_{2}\left[\frac{\partial \Omega_{0}}{\partial \mu}\right]_{\mu=\mu_{0}}+\mu_{1}\left[\frac{\partial \Omega_{1}}{\partial \mu}\right]_{\mu=\mu_{0}}+\frac{1}{2} \mu_{1}^{2}\left[\frac{\partial^{2} \Omega_{0}}{\partial \mu^{2}}\right]_{\mu=\mu_{0}}+\Omega_{2}\left(\mu_{0}\right)+\mu_{2} N}_{F_{2}}+\cdots(13
\end{aligned}
$$

The $\mu_{i} N$ term always cancels with $\mu_{i}\left[\partial \Omega_{0} / \partial \mu\right]_{\mu=\mu_{0}}$ in $F_{i}$ for $i \geq 1$ because of Eq. (10), leaving

$$
F(N)=F_{0}(N)+\underbrace{\Omega_{1}\left(\mu_{0}\right)}_{F_{1}}+\underbrace{\Omega_{2}\left(\mu_{0}\right)-\frac{1}{2} \frac{\left[\partial \Omega_{1} / \partial \mu\right]_{\mu=\mu_{0}}^{2}}{\left[\partial^{2} \Omega_{0} / \partial \mu^{2}\right]_{\mu=\mu_{0}}}}_{F_{2}}+\cdots
$$

where we've also used Eq. (12) to simplify $F_{2}$. The expansion for $F$ can be extended systematically, but this is all we need here. (Higher orders can be found by following the prescription in Refs. [46, 47].)

This construction is rather general. The Kohn-Luttinger-Ward theorem explores a particular case, the $T \rightarrow 0$ limit, in which the second term in $F_{2}$ in Eq. (14) cancels precisely against the anomalous diagram in $\Omega_{2}$, as illustrated in Fig. 2, This cancellation of derivative terms and anomalous diagrams occurs to all orders in the expansion. (An analogous cancellation was noted in the Kohn-Sham density functional (DFT) inversion in Ref. [9] 


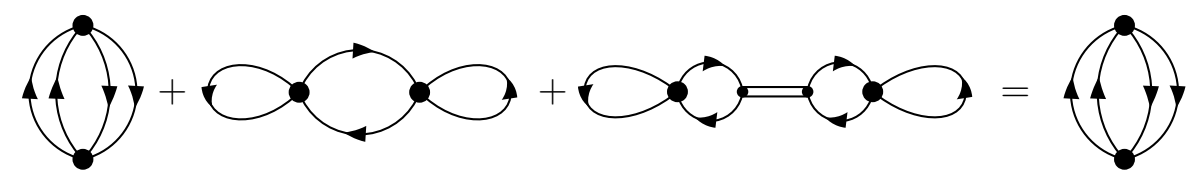

FIG. 2: Cancellation of the anomalous diagram at NLO. The double lines represents the inverse of $\left[\partial^{2} \Omega_{0} / \partial \mu^{2}\right]_{\mu=\mu_{0}}$ in Eq. (14).

and appears again in Sec. IIIA.) The end result is an expression for the free-energy $F(N)$ in terms of the diagrams used for $\Omega_{i}(\mu)$, only evaluated with $\mu=\mu_{0}$ and excluding the anomalous diagrams (both of which simplify the evaluation of $F(N)$ !). This is precisely the formalism used in Ref. [7] for a uniform low-density Fermi gas at zero temperature, where $\mu_{0}$ appeared as the Fermi energy of Eq. (11).

If we think of $\mu$ as an external (constant) source coupled to the fermion number, we can also imagine having sources coupled to other quantities and making analogous inversions. The generalization to multiple chemical potentials (i.e., constant sources coupled to conserved charges) is straightforward but adds nothing new. There are, however, two more interesting generalizations:

1. Add a second "chemical potential" $j$ coupled to a non-conserved charge, with $j$ equal to zero in the ground state. The same inversion method holds, but since we don't have a conserved charge such as $N$, we can't determine $j_{0}$ from the analog to Eq. (10). Instead, we use $j_{0}+j_{1}+j_{2}+\cdots=0$ in the ground state to solve for $j_{0}$ iteratively. (That is, guess $j_{0}$, calculate $j_{i}$ for $i>0$ in terms of $j_{0}$, find a new $j_{0}$ from $j_{0}=-\left(j_{1}+j_{2}+\cdots\right)$, and repeat to self-consistency.) It is this method that we use here to incorporate pairing for a uniform system with no external fields.

2. Add a spatially dependent (classical) source $J(\mathbf{x})$ coupled to the density operator $\psi^{\dagger} \psi$, which has expectation value $\rho(\mathbf{x}) \equiv\left\langle\psi^{\dagger} \psi\right\rangle$. Then construct the functional Legendre transformation $F[\rho]=\Omega[J]-\int \rho J$, where $J(\mathbf{x})=\delta F[\rho] / \delta \rho(\mathbf{x}) \rightarrow 0$ in the ground state. This leads to the Kohn-Sham DFT discussed in Ref. [9], where $F[\rho]$ is proportional to the effective action $\Gamma[\rho]$ for the composite operator $\psi^{\dagger} \psi$.

In Ref. [10], a spatially dependent source coupled to the kinetic energy density is included; in this case the inversion method leads to a generalization of conventional Kohn-Sham DFT that is analogous to the Skyrme-Hartree-Fock energy functional [48]. A natural generalization of Skyrme-Hartree-Fock-Bogoliubov will follow by extending the present discussion to $J(\mathbf{x})$ in finite systems.

\section{Renormalization}

Starting with $W_{2}[\mu]$, the diagrams in Fig. 1 may have ultraviolet divergences. In Ref. [7], these divergences were regulated and renormalized using dimensional regularization with minimal subtraction (DR/MS). This choice provided a clean factorization of each diagram, with the dependence on the effective range parameters solely in the coefficients and the integrals depending on the Fermi momentum $k_{\mathrm{F}}$ times universal geometric factors. The result is a very systematic power counting, with each diagram contributing to exactly one order in the EFT expansion. 
However, while DR/MS is convenient and efficient and correct, it can obscure the renormalization process. In the present case, since there are subtle questions about renormalizing effective actions with composite operators, we adopt the power divergence subtraction (PDS) scheme of Kaplan, Savage, and Wise [49], which is a generalization of DR/MS that was introduced to provide a consistent power counting in the two-body problem when the scattering length $a_{s}$ is unnaturally large [49, 50]. (An alternative for that problem is a momentum subtraction scheme proposed by Gegelia [51].) A parameter is introduced, which we call $\Lambda$ to avoid confusion with the chemical potential (this same parameter is called $\mu$ in Ref. [49]), as part of the finite subtraction. To apply PDS for natural scattering lengths, we take $\Lambda \ll 1 / a_{s}$ and expand consistently for small $\Lambda$. The DR/MS scheme is recovered by taking $\Lambda=0$. The point of keeping $\Lambda$ explicit is that we can verify that we have successfully renormalized (to a given order) by observing the cancellation of $\Lambda$ dependence between the couplings and loop integrals.

For example, consider Fig. 1(b) evaluated with $\mu_{0}$. After applying zero temperature Feynman rules, carrying out the frequency integrals, and transforming to center-of-mass variables, we obtain (see Ref. [7] for further details):

$$
\begin{aligned}
& \mathcal{E}_{2}=4 C_{0}^{2} M \nu(\nu-1) \int \frac{d^{3} s}{(2 \pi)^{3}} \int \frac{d^{3} t}{(2 \pi)^{3}}\left(\frac{\Lambda}{2}\right)^{3-D} \int \frac{d^{D} u}{(2 \pi)^{D}} \theta\left(k_{\mathrm{F}}-|\mathbf{s}+\mathbf{t}|\right) \theta\left(k_{\mathrm{F}}-|\mathbf{s}-\mathbf{t}|\right) \\
& \times\left[1-\theta\left(k_{\mathrm{F}}-|\mathbf{s}+\mathbf{u}|\right)\right]\left[1-\theta\left(k_{\mathrm{F}}-|\mathbf{s}-\mathbf{u}|\right)\right] \frac{1}{t^{2}-u^{2}+i \epsilon}
\end{aligned}
$$

with $\nu=2$ the spin degeneracy. The integral over $\mathbf{u}$ in Eq. (15), which is regulated in $D$ spatial dimensions, contains a linearly divergent term for $D=3$ coming from the piece of the integrand without any $\theta\left(k_{\mathrm{F}}-|\mathbf{s} \pm \mathbf{u}|\right)$ factors. In PDS, with the conventions of Ref. [49], this integral is:

$$
\left(\frac{\Lambda}{2}\right)^{3-D} \int \frac{d^{D} u}{(2 \pi)^{D}} \frac{1}{t^{2}-u^{2}+i \epsilon} \stackrel{\mathrm{PDS}}{\longrightarrow}-\frac{1}{4 \pi}(\Lambda+i t) .
$$

The imaginary part cancels with an equal and opposite imaginary part from the remainder of the (finite) integral over $u$ [identified using $1 /\left(t^{2}-u^{2}+i \epsilon\right)=\mathcal{P}\left\{1 /\left(t^{2}-u^{2}\right)\right\}-i \pi \delta\left(t^{2}-u^{2}\right)$ ], so that the energy density is real. The real parts of the integrals that contain $\theta\left(k_{\mathrm{F}}-|\mathbf{s} \pm \mathbf{u}|\right)$ factors reproduce the result for $\mathcal{E}_{2}$ given in Ref. [7]. The contribution proportional to $\Lambda$ is easily evaluated after changing variables to $\mathbf{q}=\mathbf{s}+\mathbf{t}$ and $\mathbf{q}^{\prime}=\mathbf{s}-\mathbf{t}$ :

$$
\begin{aligned}
\delta \mathcal{E}_{2} & =-4 C_{0}^{2} M \nu(\nu-1) \int \frac{d^{3} s}{(2 \pi)^{3}} \int \frac{d^{3} t}{(2 \pi)^{3}} \theta\left(k_{\mathrm{F}}-|\mathbf{s}+\mathbf{t}|\right) \theta\left(k_{\mathrm{F}}-|\mathbf{s}-\mathbf{t}|\right) \frac{1}{4 \pi} \Lambda \\
& =-\frac{1}{8 \pi} C_{0}^{2} M(1-1 / \nu) \Lambda\left[\nu \int^{k_{\mathrm{F}}} \frac{d^{3} q}{(2 \pi)^{3}}\right]^{2}=-\frac{1}{8 \pi} C_{0}^{2} M(1-1 / \nu) \Lambda \rho^{2},
\end{aligned}
$$

where $\rho$ is the fermion density. Thus, we have an apparent dependence in the energy on the arbitrary parameter $\Lambda$.

However, we also have a contribution at $\mathcal{O}(\Lambda)$ from the diagram in Fig. 1(a) from the dependence of $C_{0}$ on $\Lambda$ in the PDS scheme. In particular, the requirement of $\Lambda$ independence for the free-space scattering problem yields [49]:

$$
C_{0}(\Lambda)=\frac{4 \pi}{M}\left(\frac{1}{-\Lambda+1 / a_{s}}\right)=\frac{4 \pi a_{s}}{M}+\frac{4 \pi a_{s}^{2}}{M} \Lambda+\mathcal{O}\left(\Lambda^{2}\right) \equiv C_{0}^{(1)}+C_{0}^{(2)}+\cdots .
$$




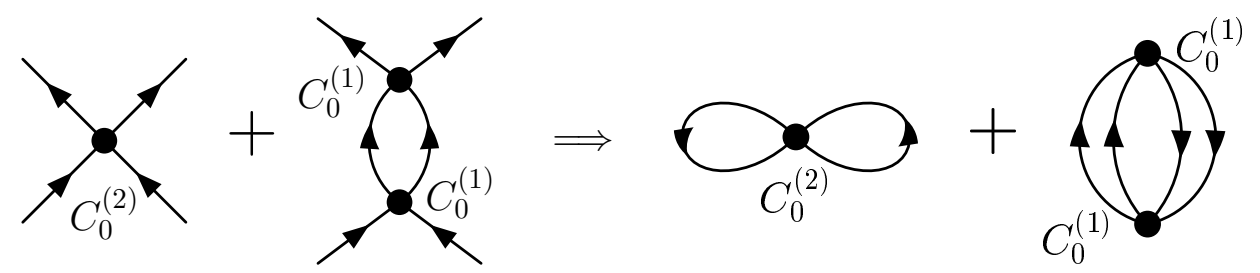

FIG. 3: Renormalization of the bubble in free space determines $C_{0}^{(2)}$ to remove the linear $\Lambda$ dependence. This implies the renormalization of the beachball (or ABA basketball [52]) diagram Fig. 1(b) at $\mathcal{O}(\Lambda)$.

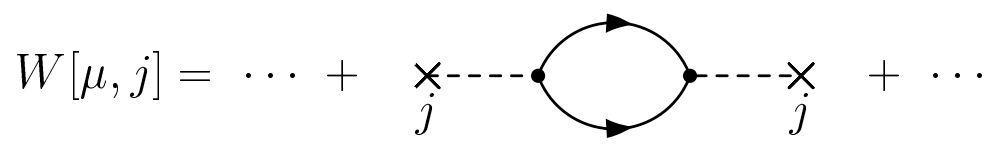

FIG. 4: Feynman diagram at second order in a perturbative expansion of $W[\mu, j]$ in $j(x)$.

If we substitute the leading (independent of $\Lambda$ ) term in Eq. (18) for $C_{0}$ in Eq. (17), we get a term proportional to $k_{\mathrm{F}}^{6} a_{s}^{2} \Lambda$. This cancels precisely with the contribution for the HartreeFock diagram Fig. 1(a) evaluated with a $C_{0}^{(2)}$ vertex (as implied by Fig. 3), which removes the linear $\Lambda$ dependence. Higher-order insertions of $C_{0}$ cancel between these diagrams and higher-order diagrams in the $k_{\mathrm{F}}$ expansion.

\section{EFFECTIVE ACTION AND INVERSION METHOD WITH PAIRING}

\section{A. Generalized Generating Functional and Inversion}

To allow the probing of pairing correlations, we extend our generating functional to include the source $j(x)$ coupled to the pair density,

$$
Z[\mu, j]=e^{-W[\mu, j]}=\int D \psi_{\alpha} D \psi_{\alpha}^{\dagger} e^{-\int d^{4} x\left\{\mathcal{L}_{E}-\mu \psi_{\alpha}^{\dagger}(x) \psi_{\alpha}(x)+j\left[\psi_{\uparrow}^{\dagger}(x) \psi_{\downarrow}^{\dagger}(x)+\psi_{\downarrow}(x) \psi_{\uparrow}(x)\right]+\frac{1}{2} \zeta j^{2}\right\}} .
$$

In general we should include both $j(x)$ and $j^{*}(x)$, but in the present case it suffices to consider real and constant $j$. The Grassmann sources $\eta$ and $\eta^{\dagger}$ are not shown, but they can be added to generate a perturbative (i.e., diagrammatic) expansion for $Z$ or $W$.

As discussed in Ref. [53] (see also Verschelde et al. [33, 34, 35]), to make finite a generating functional with a source coupled to a local composite operator, we will need to add as counterterms the most general linear combination of vertices of non-positive dimension made from that operator and the source. A perturbative expansion of $W$ in powers of $j$ immediately reveals a divergence, shown diagrammatically in Fig. 4. To renormalize in the present case, we include the term $\frac{1}{2} \zeta j^{2}$, which plays a role in the renormalization (see below) analogous to the quadratic charged scalar term in the auxiliary field approach [15, 16]. We note that this term is also the source of potential problems with the interpretation of the effective action [32]; we comment further on this issue in Sect. IV. Other counterterms in Eq. (19), including one proportional to $j\left(\psi_{\uparrow}^{\dagger} \psi_{\downarrow}^{\dagger}+\psi_{\downarrow} \psi_{\uparrow}\right)$, are implicit. 
The generating functional implies that for constant $j$ and a uniform system,

$$
\begin{aligned}
\rho & \equiv\left\langle\psi^{\dagger} \psi\right\rangle_{\mu, j}=-\frac{1}{\beta V} \frac{\partial W[\mu, j]}{\partial \mu}, \\
\phi & \equiv\left\langle\psi_{\uparrow}^{\dagger} \psi_{\downarrow}^{\dagger}+\psi_{\downarrow} \psi_{\uparrow}+\zeta j\right\rangle_{\mu, j}=\frac{1}{\beta V} \frac{\partial W[\mu, j]}{\partial j} .
\end{aligned}
$$

Note that $\phi$ corresponds to the usual definition in the ground state, where $j=0$. The explicit dependence of $\phi$ on $j$ through $\zeta$ is a consequence of the need to renormalize $\phi$ [33, 34, 35]. We generalize the KLW derivation from Sect. IIB by carrying out a double Legendre transformation, making the associations

$$
F \rightarrow \frac{1}{\beta} \Gamma \quad \text { and } \quad \Omega \rightarrow \frac{1}{\beta} W
$$

to switch variables to $\rho$ and $\phi$,

$$
\frac{1}{\beta V} \Gamma[\rho, \phi]=\frac{1}{\beta V} W[\mu, j]+\mu \rho-j \phi .
$$

We proceed in analogy to Eqs. (66) -(8) to construct the effective action $\Gamma[\rho, \phi]$ using the inversion method. In particular, we now have the expansions

$$
\begin{aligned}
W[\mu, j] & =W_{0}[\mu, j]+W_{1}[\mu, j]+W_{2}[\mu, j]+\cdots \\
\mu & =\mu_{0}+\mu_{1}+\mu_{2}+\cdots \\
j & =j_{0}+j_{1}+j_{2}+\cdots \\
\Gamma[\mu, j] & =\Gamma_{0}[\mu, j]+\Gamma_{1}[\mu, j]+\Gamma_{2}[\mu, j]+\cdots
\end{aligned}
$$

As before, the inversion is carried out by matching Eqs. (20), (21) and (23) order by order using (24)-(27), with both $\rho$ and $\phi$ counted formally as zeroth order. A complication in carrying this out is that $\phi$ will in general be renormalized order by order, which implies contributions to the left side of Eq. (21) beyond zeroth order. To avoid this, we will use the freedom in the renormalization conditions to require that there are no corrections to $\phi$, i.e., that $\phi$ and $\rho$ are given exactly by the zeroth-order equations,

$$
\rho=-\frac{1}{\beta V} \frac{\partial W_{0}\left[\mu_{0}, j_{0}\right]}{\partial \mu_{0}}, \quad \phi=\frac{1}{\beta V} \frac{\partial W_{0}\left[\mu_{0}, j_{0}\right]}{\partial j_{0}},
$$

where it is implied that $\mu_{0}$ and $j_{0}$ are determined as functions of $\rho$ and $\phi$. The contributions to $\Gamma_{i}$ from $\mu_{i} \rho$ and $j_{i} \phi$ when $i \geq 1$ cancel as in Eqs. (13) and (14) after using Eq. (28). The anomalous diagrams in $W_{2}$ cancel as in Eq. (14) and Fig. 2 against additional terms from the inversion [9, 10], leaving (with $\bar{W}_{2}$ denoting the non-anomalous part)

$$
\Gamma[\rho, \phi]=\Gamma_{0}[\rho, \phi]+\underbrace{W_{1}\left[\mu_{0}, j_{0}\right]}_{\Gamma_{1}}+\underbrace{\bar{W}_{2}\left[\mu_{0}, j_{0}\right]}_{\Gamma_{2}}+\cdots .
$$

Thus, in practice we only calculate the non-anomalous diagrams to this order.

To carry out a calculation, we truncate the expansion at a given order, construct the $W_{i}$ needed in the corresponding truncated version of Eq. (29), and determine the $\mu_{i}$ and $j_{i}$ for $i \geq 1$ from

$$
\mu_{i}=\frac{1}{\beta V} \frac{\partial \Gamma_{i}[\rho, \phi]}{\partial \rho}, \quad j_{i}=-\frac{1}{\beta V} \frac{\partial \Gamma_{i}[\rho, \phi]}{\partial \phi} .
$$




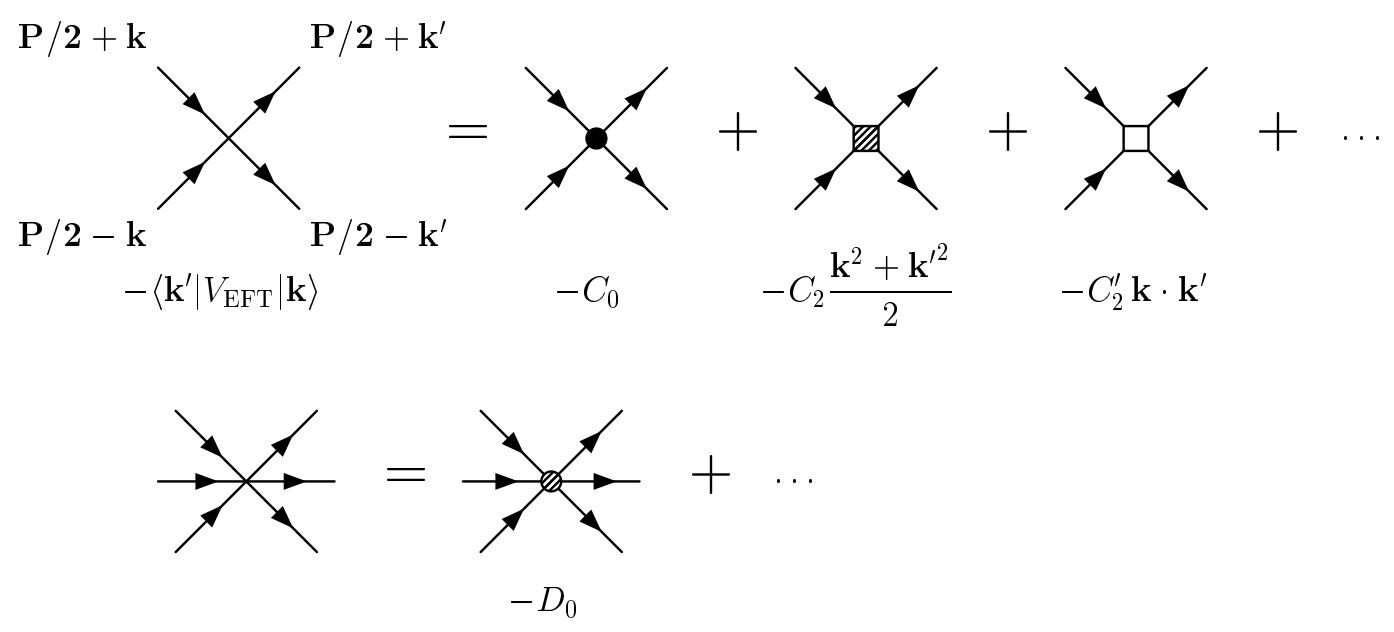

FIG. 5: Feynman rules for $-\left\langle\mathbf{k}^{\prime}\left|V_{\mathrm{EFT}}\right| \mathbf{k}\right\rangle$ and the leading 3-body contact interaction. The spin indices have been suppressed.

The values $\mu_{0}$ and $j_{0}$ are fixed by requiring both that a specified $\rho$ to be reproduced by Eq. (28) and that $j=0$, which means

$$
j_{0}=-\left(j_{1}+j_{2}+\cdots\right) .
$$

In general, these conditions can only be established numerically.

\section{B. $W_{0}[\mu, j]$ and $\Gamma_{0}[\rho, \phi]$}

To find $W_{0}$ and $\Gamma_{0}$, we need to solve a non-interacting system in the presence of external sources $\mu_{0}$ and $j_{0}$. The path integral expression for $W_{0}\left[\mu_{0}, j_{0}\right]$ with constant sources is

$$
Z_{0}\left[\mu_{0}, j_{0}\right]=e^{-W_{0}\left[\mu_{0}, j_{0}\right]}=\int D \psi_{\alpha} D \psi_{\alpha}^{\dagger} e^{-\int d^{4} x\left[\psi_{\alpha}^{\dagger}\left(\partial / \partial \tau-\nabla^{2} / 2 M-\mu_{0}\right) \psi_{\alpha}+j_{0}\left(\psi_{\uparrow} \psi_{\downarrow}+\psi_{\downarrow}^{\dagger} \psi_{\uparrow}^{\dagger}\right)+\frac{1}{2} \zeta^{(0)} j_{0}^{2}\right]}
$$

where we restrict the $\alpha$ sum to run over spin up and spin down and the $x$ dependence of the $\psi$ fields is implicit. We denote the value of $\zeta$ at this order as $\zeta^{(0)}$, which should depend on $\Lambda$ only. Since the path integral is quadratic in the fermion fields, it can be evaluated exactly in the Nambu-Gorkov formalism as $\operatorname{Tr} \ln \left(\mathbf{G}_{\mathrm{ks}}^{-1}\right)$ (see, for example, Refs. [15, 16]), where $\mathbf{G}_{\mathrm{ks}}^{-1}$ is the quadratic part of the Lagrangian and $\mathbf{G}_{\mathrm{ks}}$ is the associated Kohn-Sham Green's function. Alternatively, a canonical Bogoliubov transformation solves the problem exactly and is particularly transparent and familiar. (In a finite system, we would solve the zeroth-order system by solving Hartree-Fock-Bogoliubov equations with external KohnSham sources corresponding to a spatially dependent chemical potential and a spatially dependent pair density.) This is carried out in Appendix $\mathrm{A}$ and we simply quote that result for $W_{0}$ :

$$
\frac{1}{\beta V} W_{0}\left[\mu_{0}, j_{0}\right]=\int \frac{d^{3} k}{(2 \pi)^{3}}\left(\xi_{k}-E_{k}\right)+\frac{1}{2} \zeta^{(0)} j_{0}^{2},
$$

where

$$
\xi_{k} \equiv \varepsilon_{\mathbf{k}}^{0}-\mu_{0}
$$


with $\varepsilon_{\mathbf{k}}^{0} \equiv k^{2} / 2 M$ and

$$
E_{k} \equiv \sqrt{\xi_{k}^{2}+j_{0}^{2}}
$$

The zeroth-order effective action is then

$$
\frac{1}{\beta V} \Gamma_{0}=\frac{1}{\beta V} W_{0}+\mu_{0} \rho-j_{0} \phi .
$$

Equations for $\rho$ and $\phi$ follow from $W_{0}$ :

$$
\rho=\frac{N}{V}=-\frac{1}{\beta V} \frac{\partial W_{0}}{\partial \mu_{0}}=\int \frac{d^{3} k}{(2 \pi)^{3}}\left(1-\frac{\xi_{k}}{E_{k}}\right),
$$

and

$$
\phi=\frac{1}{\beta V} \frac{\partial W_{0}}{\partial j_{0}}=-\int \frac{d^{3} k}{(2 \pi)^{3}} \frac{j_{0}}{E_{k}}+\zeta^{(0)} j_{0} \equiv \phi_{\mathrm{B}}+\zeta^{(0)} j_{0} .
$$

Except for the extra term proportional to $\zeta^{(0)}$, these results are the same as from standard BCS calculations, but with $j_{0}$ appearing in the place of a constant gap. This is illustrated further in the left panel of Fig. 9, which plots quantities defined in Eq. (A14). With those standard definitions, $\rho=\frac{1}{2 \pi^{3}} \int d^{3} k v_{k}^{2}$ and $\phi_{\mathrm{B}}=\frac{1}{2 \pi^{3}} \int d^{3} k u_{k} v_{k}$.

The integral on the right side of Eq. (38), which we have defined as the "bare" pairing density $\phi_{\mathrm{B}}$, is linearly divergent (see the right panel of Fig. 9). This requires regularization and then renormalization through an appropriate choice of $\zeta^{(0)}$. To apply the DR/PDS scheme, we have adopted and extended the integrals derived for DR/MS in Ref. [40] in Appendix B. From Eq. (B6) we have immediately that

$$
\phi=-j_{0} I(0)+\zeta^{(0)} j_{0} .
$$

Since the interaction (i.e., $C_{0}$ ) should not appear explicitly in this zeroth-order equation, requiring $\phi$ to be the full renormalized result independent of $\Lambda$ uniquely determines

$$
\zeta^{(0)}=\frac{\Lambda M}{2 \pi}
$$

by dimensional analysis. This results in an analytic expression for $\phi$ in terms of Legendre functions [see Eqs. (B5) and (B6)]:

$$
\phi=-j_{0} \widetilde{I}(0)
$$

which is manifestly independent of $\Lambda$. Upon applying (B6) term-by-term to the density equation, we find (as expected) that the $\Lambda$ dependence cancels:

$$
\rho=\left(\frac{\Lambda}{2}\right)^{3-D} \int \frac{d^{D} k}{(2 \pi)^{D}}\left(1-\frac{\varepsilon_{\mathbf{k}}^{0}-\mu_{0}}{E_{k}}\right)=0-I(1)+\mu_{0} I(0)=-\widetilde{I}(1)+\mu_{0} \widetilde{I}(0) .
$$

By applying Eqs. (B7), these expressions for $\phi$ and $\rho$ can be expanded at small $x \equiv j_{0} / \mu_{0}$ [see Sect. [V] and Eq. (B7)]. 


\section{Feynman Rules for $W_{i}$}

The Feynman rules for the contributions to $W_{i}[\mu, j] / \beta V=\Omega_{i} / V$ from the diagrams in Fig. 1] are similar to those given in Ref. [7] (which in turn take the standard form, e.g., see Ref. [44, 45]), but with Nambu-Gorkov matrix propagators. These propagators arise from the diagonalization of $W_{0}$. The only difference from standard treatments of superconductivity (e.g., see [54]) is that $j_{0}$ appears instead of a constant gap.

The basic Feynman rules in the zero-temperature limit are as follows. First, draw all distinct, fully connected diagrams contributing to a given order in the EFT expansion of $W$. (In this paper we determine diagrams according to the $k_{\mathrm{F}}$ expansion for a dilute gas without pairing, so the power counting is the same as in Ref. [7].) Distinct diagrams are those that cannot be deformed to coincide with each other, including the direction of arrows. To evaluate a diagram:

1. Assign nonrelativistic four-momenta (frequency $k_{0}$ and three-momentum $\mathbf{k}$ ) to all lines and enforce four-momentum conservation at each vertex. At finite temperature, the frequency is a discrete Masubara fermion frequency [45].

2. For each vertex, include the corresponding term from the two-body effective potential $-\left\langle\mathbf{k}^{\prime}\left|V_{\mathrm{EFT}}\right| \mathbf{k}\right\rangle$ (or analogous three-body interaction), as shown in Fig. 5. (Recall that $\mathbf{k}$ and $\mathbf{k}^{\prime}$ are relative momenta.) The Nambu-index structure of the vertices has been suppressed in Fig. 5. For spin-independent interactions, the two-body vertices have the structure $\left[\left(\tau_{3}\right)_{\alpha \gamma}\left(\tau_{3}\right)_{\beta \delta} \pm\left(\tau_{3}\right)_{\alpha \delta}\left(\tau_{3}\right)_{\beta \gamma}\right]$, where $\alpha, \beta$ are the Nambu indices of the incoming lines and $\gamma, \delta$ are the Nambu indices of the outgoing lines. The plus sign applies to $C_{0}$ and $C_{2}$ vertices and the minus sign to $C_{2}^{\prime}$ vertices.

For each internal line include the matrix propagator $-\mathbf{G}_{0}(\widetilde{k})$, where $\widetilde{k} \equiv\left(k_{0}, \mathbf{k}\right)$ is the four-momentum assigned to the line, and

$$
\mathbf{G}_{0}(\widetilde{k})=\left(\begin{array}{cc}
\mathcal{G}_{0}\left(k_{0}, \mathbf{k}\right) & \mathcal{F}_{0}\left(k_{0}, \mathbf{k}\right) \\
\mathcal{F}_{0}^{\dagger}\left(k_{0}, \mathbf{k}\right) & -\mathcal{G}_{0}\left(-k_{0}, \mathbf{k}\right)
\end{array}\right) \equiv\left(\begin{array}{cc}
\mathcal{G}_{0 \widetilde{k}} & \mathcal{F}_{0 \widetilde{k}} \\
\mathcal{F}_{0 \widetilde{k}}^{\dagger} & -\widetilde{\mathcal{G}}_{0 \widetilde{k}}
\end{array}\right)
$$

with

$$
\mathcal{G}_{0}(\widetilde{k})=\frac{u_{k}^{2}}{i k_{0}-E_{k}}+\frac{v_{k}^{2}}{i k_{0}+E_{k}}=\frac{1}{2 E_{k}}\left[\frac{E_{k}+\xi_{k}}{i k_{0}-E_{k}}+\frac{E_{k}-\xi_{k}}{i k_{0}+E_{k}}\right]
$$

and

$$
\mathcal{F}_{0}(\widetilde{k})=\mathcal{F}_{0}^{\dagger}(\widetilde{k})=-u_{k} v_{k}\left[\frac{1}{i k_{0}-E_{k}}-\frac{1}{i k_{0}+E_{k}}\right]=\frac{j_{0}}{2 E_{k}}\left[\frac{1}{i k_{0}-E_{k}}-\frac{1}{i k_{0}+E_{k}}\right] .
$$

(Note: These conventions agree with those in Refs. [45] and [54] and differ by a sign from those in Ref. [44].) The $u_{k}$ 's and $v_{k}$ 's are defined in the Appendix in Eq. (A14) and are seen here to correspond to the residues at the poles of the noninteracting Green's functions.

3. Perform summations on Nambu indices in the diagram (e.g., do the matrix multiplication). Include -1 for every closed fermion loop (i.e., for each trace).

4. Integrate over all independent momenta with a factor $\int d^{4} k /(2 \pi)^{4}$ where $d^{4} k \equiv d k_{0} d^{3} k$. [At finite temperature, the integral $\int d k_{0} / 2 \pi$ is replaced by the sum $1 / \beta \sum_{n}$ over 
fermion frequencies $\omega_{n}$ [45].] If a spatial integral is divergent, it is defined in $D$ spatial dimensions and renormalized using the PDS subtraction scheme as discussed in Ref. [49]. For lines originating and ending at the same vertex, multiply by $\exp \left( \pm i k_{0} \eta\right)$ and take the limit $\eta \rightarrow 0^{+}$after the contour integrals have been carried out. Use $\exp \left(i k_{0} \eta\right)$ for $\mathcal{G}_{0 \widetilde{k}}$ and $\mathcal{F}_{0 \widetilde{k}}$ and $\exp \left(-i k_{0} \eta\right)$ for $\widetilde{\mathcal{G}}_{0 \widetilde{k}}$. This procedure ensures that the correct term in the Green's function is picked up.

5. Multiply by a symmetry factor $-1 /\left(S \prod_{l=2}^{l_{\max }}(l !)^{m}\right)$ where $S$ is the number of vertex permutations that transform the diagram into itself, and $m$ is the number of equivalent $l$-tuples of lines. Equivalent lines are lines that begin and end at the same vertices with the same direction of arrows.

\section{RENORMALIZATION TO LEADING ORDER}

In this section, we carry out the inversion procedure to leading order (LO), which means calculating $\Gamma_{1}[\rho, \phi]$ and determining equations for $\mu_{0}$ and $j_{0}$. We will work here in the zerotemperature limit, with frequency integrals rather than frequency sums. The final results are well known, although to our knowledge this precise formalism has not been used.

Since $\Gamma_{1}[\rho, \phi]=W_{1}\left[\mu_{0}[\rho], j_{0}[\phi]\right]$ (see Ref. [9]), we first find $W_{1}\left[\mu_{0}, j_{0}\right]$ by applying the Feynman rules to the diagram in Fig. 1(a). The overall symmetry factor is $1 / 2$ (one equivalent pair of lines), so the expression is (after cancelling signs),

$$
\begin{aligned}
& \frac{1}{\beta V} W_{1}\left[\mu_{0}, j_{0}\right]=\frac{1}{2} C_{0}^{(1)}\left[\left(\tau_{3}\right)_{\alpha \gamma}\left(\tau_{3}\right)_{\beta \delta}+\left(\tau_{3}\right)_{\alpha \delta}\left(\tau_{3}\right)_{\beta \gamma}\right] \\
& \times \int \frac{d^{4} k}{(2 \pi)^{4}} \int \frac{d^{4} k^{\prime}}{(2 \pi)^{4}} \mathbf{G}_{0}(\widetilde{p})_{\gamma \alpha} \mathbf{G}_{0}\left(\widetilde{p}^{\prime}\right)_{\delta \beta}+\frac{1}{2} \zeta^{(1)} j_{0}^{2} \\
& =\frac{1}{2} C_{0}^{(1)} \int \frac{d^{4} k}{(2 \pi)^{4}} \int \frac{d^{4} k^{\prime}}{(2 \pi)^{4}}\left[\left(\mathcal{G}_{0 \widetilde{k}}+\widetilde{\mathcal{G}}_{0 \widetilde{k}}\right)\left(\mathcal{G}_{0 \widetilde{k}^{\prime}}+\widetilde{\mathcal{G}}_{\widetilde{k}^{\prime}}\right)\right. \\
& \left.-\left(\mathcal{G}_{0 \widetilde{k}} \mathcal{G}_{0 \widetilde{k}^{\prime}}-\mathcal{F}_{0 \widetilde{k}}^{\dagger} \mathcal{F}_{0 \widetilde{k}^{\prime}}+\widetilde{\mathcal{G}}_{0 \widetilde{k}} \widetilde{\mathcal{G}}_{0 \widetilde{k}^{\prime}}-\mathcal{F}_{0 \widetilde{k}}^{\dagger} \mathcal{F}_{0 \widetilde{k}^{\prime}}\right)\right]+\frac{1}{2} \zeta^{(1)} j_{0}^{2} \\
& =C_{0}^{(1)} \int \frac{d^{4} k}{(2 \pi)^{4}} \int \frac{d^{4} k^{\prime}}{(2 \pi)^{4}}\left[\mathcal{G}_{0 \widetilde{k}} \widetilde{\mathcal{G}}_{0 \widetilde{k}^{\prime}}+\mathcal{F}_{0 \widetilde{k}}^{\dagger} \mathcal{F}_{0 \widetilde{k}^{\prime}}\right]+\frac{1}{2} \zeta^{(1)} j_{0}^{2},
\end{aligned}
$$

where we've suppressed the $e^{ \pm i k_{0} \eta}$ convergence factors and (except for $\zeta^{(1)}$ ) additional counterterm corrections discussed below. The individual integrals we need are:

$$
\begin{aligned}
\int \frac{d^{4} k}{(2 \pi)^{4}} \mathcal{G}_{0}\left( \pm k_{0}, \mathbf{k}\right) e^{ \pm i k_{0} \eta} & =\int \frac{d^{3} k}{(2 \pi)^{3}} v_{k}^{2}=\int \frac{d^{3} k}{(2 \pi)^{3}} \frac{E_{k}-\xi_{k}}{2 E_{k}}=\frac{1}{2} \rho \\
\int \frac{d^{4} k}{(2 \pi)^{4}} \mathcal{F}_{0}\left(k_{0}, \mathbf{k}\right) e^{i k_{0} \eta} & =\int \frac{d^{3} k}{(2 \pi)^{3}} u_{k} v_{k}=-j_{0}\left(\frac{\Lambda}{2}\right)^{3-D} \int \frac{d^{D} k}{(2 \pi)^{D}} \frac{1}{2 E_{k}} \equiv \frac{1}{2} \phi_{\mathrm{B}}
\end{aligned}
$$

(and $\mathcal{F}_{0}=\mathcal{F}_{0}^{\dagger}$ ). The integral in Eq. (48) has a linear divergence, which we've regulated in $D$ spatial dimensions (and identified $\phi_{\mathrm{B}}$ accordingly).

In addition to the $\frac{1}{2} \zeta^{(1)} j_{0}^{2}$ counterterm in Eq. (46), there is a counterterm $\delta Z_{j}^{(1)} j\left(\psi_{\uparrow}^{\dagger} \psi_{\downarrow}^{\dagger}+\right.$ $\psi_{\downarrow} \psi_{\uparrow}$ ), which contributes to $W_{1}$ a term proportional to $j_{0} \phi_{\mathrm{B}}$ (see Fig. 6). Our renormalization 


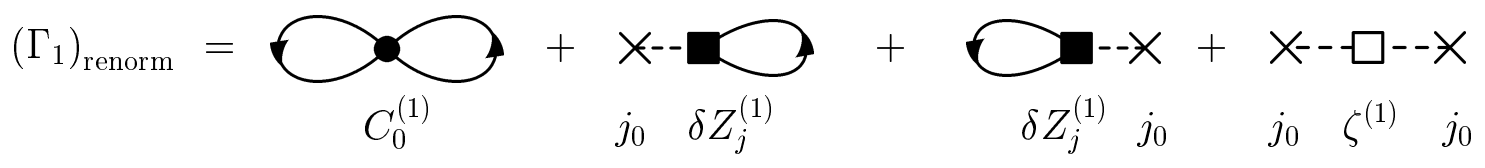

FIG. 6: Diagrammatic representation of the renormalization of $\Gamma_{1}$.

prescription is to choose this counterterm and $\zeta^{(1)}$ so that we convert $\phi_{\mathrm{B}}$ to the renormalized $\phi$ from Eq. (38) [with $\zeta^{(0)}$ from Eq. (40)]. This takes $\Gamma_{1}=W_{1}$ from a function of $\rho$ and the bare $\phi_{\mathrm{B}}$ to a function of $\rho$ and the renormalized $\phi$. This prescription fixes the counterterms and is analogous to that described for a 1PI effective action in Sect. 11.4 of Ref. [13] for tadpoles. Since the end result is simply the replacement $\phi_{\mathrm{B}} \rightarrow \phi$, there is no need to consider the explicit counterterms. We return below to the issue of additional finite contributions to $\zeta$ at this order.

This renormalization prescription allows us to write $W_{1}$ in Eq. (46) directly in terms of $\rho$ and $\phi$, yielding immediately the renormalized expression for $\Gamma_{1}$ :

$$
\frac{1}{\beta V} \Gamma_{1}[\rho, \phi]=\frac{1}{4} C_{0}^{(1)} \rho^{2}+\frac{1}{4} C_{0}^{(1)} \phi^{2} .
$$

With this result, we can evaluate $\mu_{1}$ and $j_{1}$ directly:

$$
\mu_{1}=\frac{1}{\beta V} \frac{\partial \Gamma_{1}}{\partial \rho}=\frac{1}{2} C_{0}^{(1)} \rho
$$

and

$$
j_{1}=-\frac{1}{\beta V} \frac{\partial \Gamma_{1}}{\partial \phi}=-\frac{1}{2} C_{0}^{(1)} \phi .
$$

More generally, we will need to eliminate $\mu_{0}$ and $j_{0}$ from the $W_{i}$ in favor of $\rho$ and $\phi$ in order to construct the $\Gamma_{i}$. Since the particle number (and hence the density for a uniform system) is given, the correction $\mu_{1}$ is known. In contrast, the pairing density $\phi$, which determines $j_{1}$, is not fixed by Eq. (38) unless $j_{0}$ (and $\mu_{0}$ ) is known. But since we take $j=0$ in the ground state, and $j=j_{0}+j_{1}$ to this order, we have for the ground state that

$$
j_{0}=-j_{1}=\frac{1}{2} C_{0}^{(1)} \phi .
$$

Substituting from Eq. (38), we get the "gap equation"

$$
j_{0}=-\frac{C_{0}^{(1)}}{2}\left[\left(\frac{\Lambda}{2}\right)^{3-D} \int \frac{d^{D} k}{(2 \pi)^{D}} \frac{j_{0}}{E_{k}}-\zeta^{(0)} j_{0}\right]=-\frac{C_{0}^{(1)}}{2} j_{0}\left[I(0)-\zeta^{(0)}\right],
$$

where we've used $I(0)$ as defined in Appendix B. This has the trivial solution $j_{0}=0$ but also the possibility of a non-zero "superconducting" solution for $j_{0}$ (and therefore $\phi$ ) for $C_{0}^{(1)}<0$.

If we evaluate the gap equation in $\mathrm{DR} / \mathrm{PDS}$ using $I(0)$ from Eq. (B5) for $D=3$ and $\zeta^{(0)}$ from Eq. (40), the $\Lambda$ dependence is precisely eliminated, leaving

$$
1=-\frac{C_{0}^{(1)}}{2} \widetilde{I}(0)=-\sqrt{2 M \mu_{0}}\left|a_{s}\right|\left(1+x^{2}\right)^{1 / 4} P_{1 / 2}^{0}\left(\frac{-1}{\sqrt{1+x^{2}}}\right),
$$


with $\widetilde{I}(0)$ defined in Eq. ( $(\underline{\mathrm{B} 6})$. With $\mu_{0}=k_{\mathrm{F}}^{2} / 2 M$, this is the same gap equation derived in Ref. [40], with $j_{0}$ taking the place of the gap $\Delta$. If we use the leading term in the expansion of $\widetilde{I}(0)$ from Eq. (B7), we find

$$
x=\left|\frac{j_{0}}{\mu_{0}}\right| \approx \frac{8}{e^{2}} e^{-\pi / 2 k_{\mathrm{F}}\left|a_{s}\right|},
$$

as usual for the BCS weak-coupling limit. This is a very good approximation even for moderate values of $k_{\mathrm{F}}\left|a_{s}\right|$; in particular, the approximation for $x$ is less than the exact solution to Eq. (54) by $0.06 \%, 0.8 \%, 2.7 \%, 3.8 \%$ for $k_{\mathrm{F}}\left|a_{s}\right|=0.5,1.0,2.0,3.0$, respectively. If we expand the expression for $\rho$ in Eq. (42), we find that the $\log x$ dependence from the leading term cancels, leaving

$$
\rho=\frac{k_{\mathrm{F}}^{3}}{3 \pi^{2}}\left[1+\frac{3}{16} x^{2}\left(1-2 \log \frac{x}{8}\right)\right] .
$$

Thus we get the free Fermi gas result with a correction that depends on $x$. For $k_{\mathrm{F}}\left|a_{s}\right| \approx 0.5$, we find $x \approx 0.05$, and the correction is about $0.5 \%$.

The renormalized effective action to this order is found by combining Eqs. (36) and (49), and using the results in Appendix B:

$$
\begin{aligned}
\frac{1}{\beta V}\left(\Gamma_{0}+\Gamma_{1}\right)= & \left(\frac{\Lambda}{2}\right)^{3-D} \int \frac{d^{D} k}{(2 \pi)^{D}}\left(\xi_{k}-E_{k}\right)+\frac{1}{2} \zeta^{(0)} j_{0}^{2}+\mu_{0} \rho-j_{0} \phi+\frac{1}{4} C_{0}^{(1)} \rho^{2}+\frac{1}{4} C_{0}^{(1)} \phi^{2} \\
= & 0-I(2)+2 \mu_{0} I(1)-\left(\mu_{0}^{2}+j_{0}^{2}\right) I(0)+\frac{1}{2} \frac{\Lambda M}{2 \pi} j_{0}^{2}+\mu_{0} \rho-j_{0} \phi \\
& \quad+\frac{1}{4} C_{0}^{(1)} \rho^{2}+\frac{1}{4} C_{0}^{(1)} \phi^{2} \\
= & -\widetilde{I}(2)+2 \mu_{0} \widetilde{I}(1)-\left(\mu_{0}^{2}+j_{0}^{2}\right) \widetilde{I}(0)+\mu_{0} \rho-j_{0} \phi+\frac{1}{4} C_{0}^{(1)} \rho^{2}+\frac{1}{4} C_{0}^{(1)} \phi^{2},(57)
\end{aligned}
$$

which is manifestly independent of $\Lambda$. To find the energy density, we evaluate at the stationary point:

$$
\frac{E}{V}=\left.\frac{1}{\beta V}\left(\Gamma_{0}+\Gamma_{1}\right)\right|_{j_{0}=-\frac{1}{2}\left|C_{0}^{(1)}\right| \phi}=-\widetilde{I}(2)+\mu_{0} \widetilde{I}(1)-\frac{\left|C_{0}^{(1)}\right|}{4} \rho^{2}-\frac{1}{\left|C_{0}^{(1)}\right|} \mu_{0}^{2} x^{2},
$$

where we've used Eqs. (54) and (42) to reach the final form.

In practice, however, a different renormalization can be more useful for the numerical solution of the self-consistent equations and for extending the results to inhomogeneous finite systems in the local density approximation (LDA) and to higher orders. The conventional approach to renormalizing the gap equation for a delta-function interaction is based on the observation that the unrenormalized gap equation (with a gap $\Delta$ taking the place of $j_{0}$ in $\left.E_{k}\right)$

$$
1=\frac{\left|C_{0}\right|}{2} \int \frac{d^{3} k}{(2 \pi)^{3}} \frac{1}{E_{k}}
$$

has the same linear ultraviolet divergence as the unrenormalized expression for zero-energy scattering in terms of the scattering length [39, 40],

$$
\frac{M\left|C_{0}\right|}{4 \pi a_{s}}+1=\frac{\left|C_{0}\right|}{2} \int \frac{d^{3} k}{(2 \pi)^{3}} \frac{1}{\varepsilon_{\mathbf{k}}} .
$$


Thus, one can eliminate the unrenormalized $C_{0}$ directly (e.g., by subtracting the equations and dividing out $C_{0}$ ) to obtain:

$$
\frac{M}{4 \pi a_{s}}=\frac{1}{C_{0}^{(1)}}=-\frac{1}{2} \int \frac{d^{3} k}{(2 \pi)^{3}}\left[\frac{1}{E_{k}}-\frac{1}{\varepsilon_{\mathbf{k}}}\right],
$$

which is finite and can be evaluated using elliptic integrals [39]. This same result is obtained within the present formalism by comparing Eqs. (38) and (61) and making the association:

$$
\zeta^{(0)}=\frac{\Lambda M}{2 \pi} \rightarrow \int \frac{d^{3} k}{(2 \pi)^{3}} \frac{1}{\varepsilon_{\mathbf{k}}} .
$$

This connection gives us a way to eliminate $\zeta^{(0)}$ explicitly in the energy and $\phi$ equations, leaving finite expressions that can be evaluated numerically.

Thus, the energy density from Eqs. (57) and (58) can be evaluated as

$$
\begin{aligned}
\frac{E}{V} & =\int \frac{d^{3} k}{(2 \pi)^{3}}\left[\xi_{k}-E_{k}+\frac{1}{2} \frac{j_{0}^{2}}{\varepsilon_{\mathbf{k}}}\right]+\left[\mu_{0}-\frac{1}{4}\left|C_{0}^{(1)}\right| \rho\right] \rho+\frac{j_{0}^{2}}{\left|C_{0}^{(1)}\right|} \\
& =\int \frac{d^{3} k}{(2 \pi)^{3}}\left[\xi_{k}-E_{k}+\frac{1}{2} \frac{j_{0}^{2}}{E_{k}}\right]+\left[\mu_{0}-\frac{1}{4}\left|C_{0}^{(1)}\right| \rho\right] \rho .
\end{aligned}
$$

The expressions for $\rho$ and $\phi$ in this approach are

$$
\rho=\int \frac{d^{3} k}{(2 \pi)^{3}}\left(1-\frac{\xi_{k}}{E_{k}}\right) \text { and } \phi=-j_{0} \int \frac{d^{3} k}{(2 \pi)^{3}}\left[\frac{1}{E_{k}}-\frac{1}{\varepsilon_{\mathbf{k}}}\right] .
$$

We note that the usual normal-state LO results are recovered in the $j_{0} \rightarrow 0$ limit:

$$
\frac{E}{V} \rightarrow \frac{3}{5} \mu_{0} \rho-\frac{1}{4}\left|C_{0}^{(1)}\right| \rho^{2} \quad \text { and } \quad \rho \rightarrow \frac{1}{3 \pi^{2}} k_{\mathrm{F}}^{3} \quad \text { and } \quad \mu_{0} \rightarrow \frac{k_{\mathrm{F}}^{2}}{2 M}
$$

More efficient evaluations of $\phi$ have been discussed by Bulgac and Yu [55, 56, 57] in the context of local density approximations (LDA's) for finite systems. In the present case, we simply illustrate how improvements can work by comparing the evaluation of $\phi$ for two different subtractions. We define $\phi_{E_{c}}$ as the evaluation of $\phi$ as a numerical integral up to a maximum $k_{c}$ given by $E_{c}=k_{c}^{2} / 2 M$. Then we compare the two expressions for $\phi_{E_{c}}$,

$$
\phi_{E_{c}}=-j_{0} \int^{k_{c}} \frac{d^{3} k}{(2 \pi)^{3}}\left[\frac{1}{E_{k}}-\frac{1}{\varepsilon_{\mathbf{k}}}\right]=-j_{0} \int^{k_{c}} \frac{d^{3} k}{(2 \pi)^{3}}\left[\frac{1}{E_{k}}-\frac{\mathcal{P}}{\varepsilon_{\mathbf{k}}-\mu_{0}}\right]
$$

where the subtractions are equal in the $k_{c} \rightarrow \infty$ limit. However, as shown in Fig. [7, the rate of convergence is, in practice, dramatically improved by using the second subtraction. This will be important in applications to finite systems.

The simple interpretation of the effective action as a variational energy functional is apparently altered by the requirement of sources appearing beyond linear order [32]. In particular, we recall the connection between an effective action and a variational estimate of the energy, either in Euclidean space [53] or in Minkowski space as a constrained minimization with the sources acting as Lagrange multipliers [14]. We consider the former, with a generic 


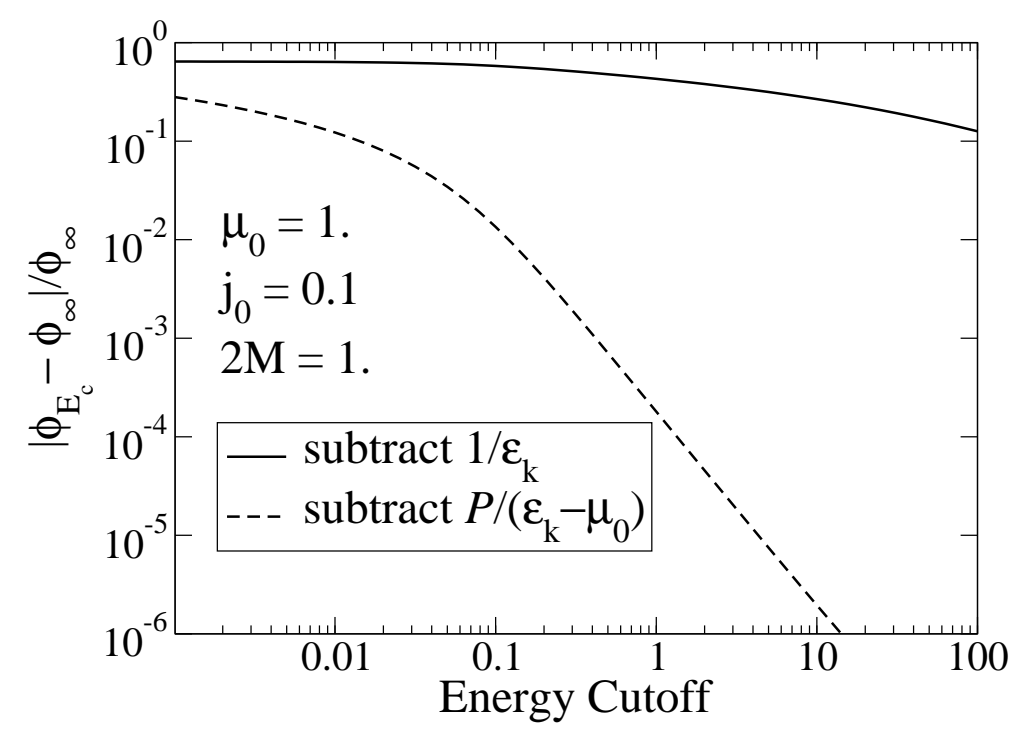

FIG. 7: Convergence of the integral for the pair density in the uniform system for two subtractions as a function of an energy cutoff $E_{c}$ in the integral. The converged value of $\phi$ is denoted $\phi_{\infty}$.

Hamiltonian $\widehat{H}$ and external source $J(\mathbf{x})$ with only spatial dependence coupled to a density $\widehat{\rho}$ (which represent vectors of sources and densities in the present case):

$$
\widehat{H}(J)=\widehat{H}+\int J \widehat{\rho}
$$

If the ground state is isolated (and bounded from below), then as $\beta \rightarrow \infty$ the partition function in the presence of $J, \mathcal{Z}[J]$, projects on the ground state of $\widehat{H}(J)$ with energy $E_{0}(J)$ :

$$
\mathcal{Z}[J]=e^{-W[J]} \sim \operatorname{Tr} e^{-\beta(\widehat{H}+J \widehat{\rho})} \Longrightarrow E_{0}(J)=\lim _{\beta \rightarrow \infty}-\frac{1}{\beta} \log \mathcal{Z}[J]=\lim _{\beta \rightarrow \infty} \frac{1}{\beta} W[J] .
$$

Thus, separating out the explicit dependence on $J$ in $\widehat{H}(J)$,

$$
E_{0}(J)=\langle\widehat{H}(J)\rangle_{J}=\langle\widehat{H}\rangle_{J}+\int J\langle\widehat{\rho}\rangle_{J},
$$

where $\langle\widehat{O}\rangle_{J}$ means the expectation value of $\widehat{O}$ in the ground state in the presence of $J$. Combining these results, the expectation value of $\widehat{H}$ in the ground state generated by $J[\rho]$ is

$$
\langle\widehat{H}\rangle_{J}=\frac{1}{\beta} W[J]-\int J \rho=\frac{1}{\beta} \Gamma[\rho] \stackrel{J \rightarrow 0}{\longrightarrow} E_{0} .
$$

Thus, we conclude that $\Gamma[\rho]$ provides a variational estimate of the energy. However, it would seem that this schematic argument breaks down if the source $J$ appears nonlinearly in Eq. (67). Similarly, the interpretation of $J$ as a generalized Lagrange multiplier relies on it appearing linearly [14]. Another alternative would be to remove nonlinear terms in favor of auxiliary fields, as discussed in Refs. [33, 34]. However, it is still the case that the ground state energy is given at a stationary point (corresponding to $J \rightarrow 0$ ), as long as the operator corresponding to $\rho$ is defined through $\rho=\delta W[J] / \delta J$. 


\section{RENORMALIZATION TO NEXT-TO-LEADING ORDER}

In this section, we extend the inversion procedure to next-to-leading order (NLO) at zero temperature, which means calculating $\Gamma_{2}\left[\mu_{0}, j_{0}\right]$ and determining equations for $\mu_{0}$ and $j_{0}$. We start with the diagrams for $W_{2}$ in Fig. 1(b) and (c) evaluated with $C_{0}^{(1)}$ vertices, together

with the $W_{1}$ diagram evaluated with the $C_{0}^{(2)}$ vertex. The latter diagram has two terms, each of which will remove a linear $\Lambda$ dependence corresponding to ultraviolet divergences from each of the two $W_{2}$ diagrams. The first term cancels a divergence and $\Lambda$ dependence from the beachball, just as in Sect. IIC. The second term cancels a new divergence in the anomalous diagram. Finally, we will have the counterterm proportional to $j\left(\psi_{\uparrow}^{\dagger} \psi_{\downarrow}^{\dagger}+\psi_{\downarrow} \psi_{\uparrow}\right)$, which is again chosen to convert $\phi_{\mathrm{B}}$ to the renormalized $\phi$ in the anomalous diagram Fig. 1(c).

Applying the Feynman rules to $W_{2}^{(b)}$ and $W_{2}^{(c)}$ and then carrying out the frequency integrals and simplifying through variable transformations, we obtain

$$
\begin{aligned}
& \frac{1}{\beta V} W_{2}^{(b)}\left[\mu_{0}, j_{0}\right]=-\frac{1}{4}\left(C_{0}^{(1)}\right)^{2} \int \frac{d^{4} p}{(2 \pi)^{4}} \int \frac{d^{4} k}{(2 \pi)^{4}} \int \frac{d^{4} q}{(2 \pi)^{4}} \\
& \times\left\{\operatorname{Tr}\left[\mathbf{G}_{0}(\widetilde{p}) \tau_{3} \mathbf{G}_{0}(\widetilde{p}-\widetilde{q}) \tau_{3}\right] \cdot \operatorname{Tr}\left[\mathbf{G}_{0}(\widetilde{k}) \tau_{3} \mathbf{G}_{0}(\widetilde{k}+\widetilde{q}) \tau_{3}\right]\right. \\
& \left.-\operatorname{Tr}\left[\mathbf{G}_{0}(\widetilde{p}) \tau_{3} \mathbf{G}_{0}(\widetilde{p}-\widetilde{q}) \tau_{3} \mathbf{G}_{0}(\widetilde{k}) \tau_{3} \mathbf{G}_{0}(\widetilde{k}+\widetilde{q}) \tau_{3}\right]\right\} \\
& =-\frac{1}{2}\left(C_{0}^{(1)}\right)^{2} \int \frac{d^{4} p}{(2 \pi)^{4}} \int \frac{d^{4} k}{(2 \pi)^{4}} \int \frac{d^{4} q}{(2 \pi)^{4}}\left[\mathcal{G}_{0 \widetilde{p}} \mathcal{G}_{0 \widetilde{p}-\widetilde{q}} \mathcal{G}_{0 \widetilde{k}} \mathcal{G}_{0 \widetilde{k}+\widetilde{q}}\right. \\
& \left.+\mathcal{F}_{0 \widetilde{p}} \mathcal{F}_{0 \widetilde{p}-\widetilde{q}} \mathcal{F}_{0 \widetilde{k}} \mathcal{F}_{0 \widetilde{k}+\widetilde{q}}+2 \mathcal{G}_{0 \widetilde{p}} \widetilde{\mathcal{G}}_{0 \widetilde{p}-\widetilde{q}} \mathcal{F}_{0 \widetilde{k}} \mathcal{F}_{0 \widetilde{k}+\widetilde{q}}\right] \\
& =-\left(C_{0}^{(1)}\right)^{2} \int \frac{d^{3} p}{(2 \pi)^{3}} \int \frac{d^{3} k}{(2 \pi)^{3}} \int \frac{d^{3} q}{(2 \pi)^{3}} \frac{1}{E_{p}+E_{k}+E_{p-q}+E_{k+q}} \text {, } \\
& \times\left[u_{p}^{2} u_{k}^{2} v_{p-q}^{2} v_{k+q}^{2}-2 u_{p}^{2} v_{k}^{2}(u v)_{p-q}(u v)_{k+q}\right. \\
& \left.+(u v)_{p}(u v)_{k}(u v)_{p-q}(u v)_{k+q}\right]
\end{aligned}
$$

and

$$
\begin{aligned}
& \frac{1}{\beta V} W_{2}^{(c)}\left[\mu_{0}, j_{0}\right]=\frac{1}{2}\left(C_{0}^{(1)}\right)^{2} \int \frac{d^{4} p}{(2 \pi)^{4}} \int \frac{d^{4} k}{(2 \pi)^{4}} \int \frac{d^{4} q}{(2 \pi)^{4}} \\
& \times\{ \operatorname{Tr}\left[\mathbf{G}_{0}(\widetilde{p}) \tau_{3}\right] \cdot \operatorname{Tr}\left[\mathbf{G}_{0}(\widetilde{k}) \tau_{3} \mathbf{G}_{0}(\widetilde{k}) \tau_{3}\right] \cdot \operatorname{Tr}\left[\mathbf{G}_{0}(\widetilde{q}) \tau_{3}\right] \\
&-2 \operatorname{Tr}\left[\mathbf{G}_{0}(\widetilde{p}) \tau_{3}\right] \cdot \operatorname{Tr}\left[\mathbf{G}_{0}(\widetilde{k}) \tau_{3} \mathbf{G}_{0}(\widetilde{k}) \tau_{3} \mathbf{G}_{0}(\widetilde{q}) \tau_{3}\right] \\
&\left.+\operatorname{Tr}\left[\mathbf{G}_{0}(\widetilde{p}) \tau_{3} \mathbf{G}_{0}(\widetilde{k}) \tau_{3} \mathbf{G}_{0}(\widetilde{q}) \tau_{3} \mathbf{G}_{0}(\widetilde{k}) \tau_{3}\right]\right\} \\
&=-\left(C_{0}^{(1)}\right)^{2} \int \frac{d^{3} k}{(2 \pi)^{3}} \frac{1}{2 E_{k}}\left[\rho\left(u_{k} v_{k}\right)^{2}+\frac{1}{2} \phi_{\mathrm{B}}\left(u_{k}^{2}-v_{k}^{2}\right)\right]^{2}
\end{aligned}
$$

We can identify potential ultraviolet divergences by noting that

$$
\begin{aligned}
& v_{k}^{2}=\frac{1}{2}\left(1-\frac{\xi_{k}}{E_{k}}\right) \stackrel{k \rightarrow \infty}{\longrightarrow} \frac{j_{0}^{2} M^{2}}{k^{4}} \\
& u_{k}^{2}=\frac{1}{2}\left(1+\frac{\xi_{k}}{E_{k}}\right) \stackrel{k \rightarrow \infty}{\longrightarrow} 1-\frac{j_{0}^{2} M^{2}}{k^{4}}
\end{aligned}
$$


a)

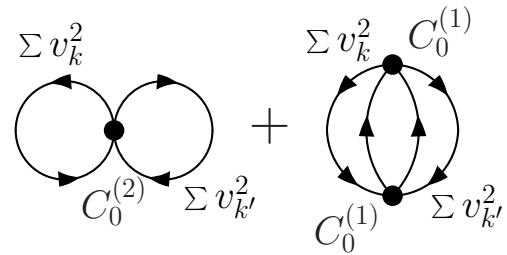

b)

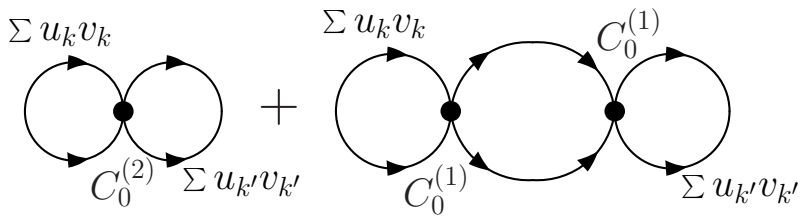

FIG. 8: Renormalization of ultraviolet divergences at NLO. The divergent parts of $W_{2}$ evaluated with $C_{0}^{(1)}$ are shown with the corresponding $W_{1}$ contributions evaluated with $C_{0}^{(2)}$, which precisely cancels the $\Lambda$ dependence.

$$
u_{k} v_{k}=-\frac{j_{0}}{2 E_{k}} \stackrel{k \rightarrow \infty}{\longrightarrow}-\frac{j_{0} M}{k^{2}}
$$

and

$$
\frac{1}{E_{k}} \stackrel{k \rightarrow \infty}{\longrightarrow} \frac{2 M}{k^{2}}
$$

Using these asymptotic behaviors, we see that in $W_{2}^{(b)}$ it is only the term with $u_{k}^{2} u_{p}^{2}$ that can be ultraviolet divergent (see Fig. 8a), while in $W_{2}^{(c)}$ it is the term with $u_{k}^{4}$ times $\phi_{\mathrm{B}}^{2}$ (see fig. $8 \mathrm{~b})$.

To isolate and cancel the divergent contribution in $W_{2}^{(b)}$, it is easiest to switch variables to $\mathbf{s}, \mathbf{t}$, and $\mathbf{u}$, where $\mathbf{p}=\mathbf{s}+\mathbf{u}, \mathbf{k}=\mathbf{s}-\mathbf{u}, \mathbf{p}-\mathbf{q}=\mathbf{s}-\mathbf{t}, \mathbf{k}+\mathbf{q}=\mathbf{s}+\mathbf{t}$, and we pick up a factor of 8 from the Jacobian. After replacing $u_{\mathbf{s} \pm \mathbf{u}}^{2}$ by $1-v_{\mathbf{s} \pm \mathbf{u}}^{2}$ and keeping only the factors with 1's, we find the divergent part

$$
\frac{1}{\beta V}\left[W_{2}^{(b)}\right]_{\text {divergent }}=-8\left(C_{0}^{(1)}\right)^{2} \int \frac{d^{3} s}{(2 \pi)^{3}} \int \frac{d^{3} t}{(2 \pi)^{3}} \int \frac{d^{3} u}{(2 \pi)^{3}} \frac{v_{\mathbf{s}-\mathbf{t}}^{2} v_{\mathbf{s}+\mathbf{t}}^{2}}{E_{\mathbf{s}+\mathbf{u}}+E_{\mathbf{s}-\mathbf{u}}+E_{\mathbf{s}+\mathbf{t}}+E_{\mathbf{s}-\mathbf{t}}} .
$$

For large $u$, we see from Eq. (76) that Eq. (77) has the same linear divergence as found for $\mathcal{E}_{2}$ in the $j_{0}=0$ limit in Sect. IIC. We stress that with the divergence regulated in DR, consistent renormalization requires that the $\Lambda$ dependence be removed by the contribution from the Hartree-Fock diagram evaluated with the $C_{0}^{(2)}$ vertex (but now with $j_{0} \neq 0$ ) without adjustment. The latter is found from the $\rho^{2}$ term in $W_{1}$ [see Eq. (46)] with $C_{0}^{(1)} \rightarrow C_{0}^{(2)}=$ $4 \pi a_{s}^{2} \Lambda / M$

$$
\frac{1}{\beta V} \delta W_{2}^{(b)}=\frac{4 \pi a_{s}^{2} \Lambda}{M} \int \frac{d^{3} k}{(2 \pi)^{3}} \int \frac{d^{3} k^{\prime}}{(2 \pi)^{3}} v_{k}^{2} v_{k}^{\prime 2} .
$$

To carry out the same sort of DR analysis as for LO, we would need to analytically continue Eq. (77) in $D$. A more practical alternative is to subtract and add the $j_{0}=0$ version of the integral (also taking $\varepsilon_{s}$ and $\varepsilon_{t}$ less than $\mu_{0}$ ), which can be evaluated using the integral in Eq. (16):

$$
\begin{aligned}
& -8\left(C_{0}^{(1)}\right)^{2} \int \frac{d^{3} s}{(2 \pi)^{3}} \int \frac{d^{3} t}{(2 \pi)^{3}}\left(\frac{\Lambda}{2}\right)^{3-D} \mathcal{P} \int \frac{d^{D} u}{(2 \pi)^{D}} \frac{v_{\mathbf{s}-\mathbf{t}}^{2} v_{\mathbf{s}+\mathbf{t}}^{2}}{\varepsilon_{\mathbf{s}+\mathbf{u}}+\varepsilon_{\mathbf{s}-\mathbf{u}}-\varepsilon_{\mathbf{s}+\mathbf{t}}-\varepsilon_{\mathbf{s}-\mathbf{t}}} \\
= & -8 M\left(C_{0}^{(1)}\right)^{2} \int \frac{d^{3} s}{(2 \pi)^{3}} \int \frac{d^{3} t}{(2 \pi)^{3}} v_{\mathbf{s}-\mathbf{t}}^{2} v_{\mathbf{s}+\mathbf{t}}^{2}\left(\frac{\Lambda}{2}\right)^{3-D} \int \frac{d^{D} u}{(2 \pi)^{D}} \frac{\mathcal{P}}{u^{2}-t^{2}} \\
= & 8 \frac{4 \pi a_{s}^{2} \Lambda}{M} \int \frac{d^{3} s}{(2 \pi)^{3}} \int \frac{d^{3} t}{(2 \pi)^{3}} v_{\mathbf{s}-\mathbf{t}}^{2} v_{\mathbf{s}+\mathbf{t}}^{2},
\end{aligned}
$$


which, after a change of variables, is seen to equal $-\frac{1}{\beta V} \delta W_{2}^{(b)}$. Thus, the renormalized version of Eq. (77) is obtained with the substitution:

$$
\frac{1}{E_{\mathbf{s}+\mathbf{u}}+E_{\mathbf{s}-\mathbf{u}}+E_{\mathbf{s}+\mathbf{t}}+E_{\mathbf{s}-\mathbf{t}}} \longrightarrow \frac{1}{E_{\mathbf{s}+\mathbf{u}}+E_{\mathbf{s}-\mathbf{u}}+E_{\mathbf{s}+\mathbf{t}}+E_{\mathbf{s}-\mathbf{t}}}-\frac{\mathcal{P}}{\varepsilon_{\mathbf{s}+\mathbf{u}}+\varepsilon_{\mathbf{s}-\mathbf{u}}-\varepsilon_{\mathbf{s}+\mathbf{t}}-\varepsilon_{\mathbf{s}-\mathbf{t}}},
$$

which makes the integral explicitly finite and suitable for numerical evaluation.

The other part of $W_{1}$ with $C_{0}^{(2)}$ renormalizes the anomalous $W_{2}$ contribution. In particular, we isolate the divergence in Eq. (72) by the replacement $u_{k}^{2} \rightarrow 1-v_{k}^{2}$. Then the divergent part is

$$
\frac{1}{\beta V}\left[W_{2}^{(c)}\right]_{\text {divergent }}=-\left(C_{0}^{(1)}\right)^{2} \int \frac{d^{3} k}{(2 \pi)^{3}} \frac{1}{2 E_{k}} \frac{1}{4} \phi_{\mathrm{B}}^{2} .
$$

In this case, the substitution

$$
\frac{1}{E_{k}} \longrightarrow \frac{1}{E_{k}}-\frac{\mathcal{P}}{\varepsilon_{\mathbf{k}}}
$$

renormalizes the anomalous $W_{2}$. Using Eq. (62), we see that the additional piece is supplied precisely by the second term in $W_{1}$ with $C_{0}^{(1)} \rightarrow C_{0}^{(2)}$, as advertised.

The prescription for deriving $\Gamma_{2}$ from $W_{2}$ was detailed in Ref. [9] for the case of a source $J(\mathbf{x})$ coupled to the density, and is reproduced for a constant chemical potential in Eq. (14). The end result is simple: the additional terms from the inversion method cancel precisely against the anomalous diagram (for zero-range forces). This same cancellation holds more generally with multiple sources and corresponding Legendre transformations (the demonstration follows directly by putting sources and densities into a vector, and is not shown here). Here one has to check that the counterterm contributions are consistent with this cancellation. After the removal of the anomalous diagram $W_{2}^{(c)}$, the evaluation of $\Gamma_{2}[\rho, \phi]$ reduces to evaluating the renormalized beachball diagram. That is, $\Gamma_{2}$ is given by Eq. (71) with the term corresponding to Eq. (77) evaluated with the substitution in Eq. (80).

While the NLO result for the energy follows formally as at LO, in practice the solution does not follow as directly. It particular, analytic expressions are not available for the $\Gamma_{2}$ integrals and $j_{2}=-(1 / \beta V) \partial \Gamma_{2} /\left.\partial \phi\right|_{\rho}$ is not simply proportional to $\phi$ (unlike $j_{1}$ ), and so $j_{0}=-j_{1}-j_{2}$ does not yield a simple gap equation. A possible solution method is to directly solve $\partial\left[\Gamma_{0}+\Gamma_{1}+\Gamma_{2}\right] /\left.\partial \phi\right|_{\rho}=0$, for example via a steepest descent method, by varying $\mu_{0}$ and $j_{0}$ while keeping $\rho$ constant using Eq. (42). This task can be simplified by expanding about the LO solution and/or using the expansions for small $x=j_{0} / \mu_{0}$. Such numerical solutions have yet to be investigated.

Although the full result for $\Gamma_{2}[\rho, \phi]$ is only accessible numerically, we can extract the leading correction to the gap equation analytically, which at NLO results in a universal (i.e., independent of $a_{s}$ ) change in the prefactor of $j_{0}$ (which we associate with the gap). This contribution can be extracted by isolating the contribution to $\Gamma_{2}$ that has the logarithmic divergence at the Fermi surface of the same form as in $W_{1}$, which means two factors of $u v$. We can identify this part in $W_{2}^{(b)}$ by letting $\mathbf{p}-\mathbf{q} \rightarrow \mathbf{k}$ and $\mathbf{k}+\mathbf{q} \rightarrow \mathbf{k}^{\prime}$ in $\Gamma_{2}$ to obtain

$$
\frac{1}{\beta V}\left[\Gamma_{2}\right]_{\text {induced }}=2\left(C_{0}^{(1)}\right)^{2} \int \frac{d^{3} k}{(2 \pi)^{3}} u_{k} v_{k} \int \frac{d^{3} k^{\prime}}{(2 \pi)^{3}} u_{k^{\prime}} v_{k^{\prime}} \int \frac{d^{3} q}{(2 \pi)^{3}} \frac{u_{\mathbf{k}+\mathbf{q}}^{2} v_{\mathbf{k}^{\prime}-\mathbf{q}}^{2}}{E_{\mathbf{k}}+E_{\mathbf{k}^{\prime}}+E_{\mathbf{k}+\mathbf{q}}+E_{\mathbf{k}^{\prime}-\mathbf{q}}} .
$$

We note that the terms proportional to $u_{k} v_{k}$ peak at $\mu_{0}$ as $j_{0} \rightarrow 0$ (see Fig. 9). To extract the leading contribution from the log divergence at the Fermi surface, we can set $j_{0}=0$ 

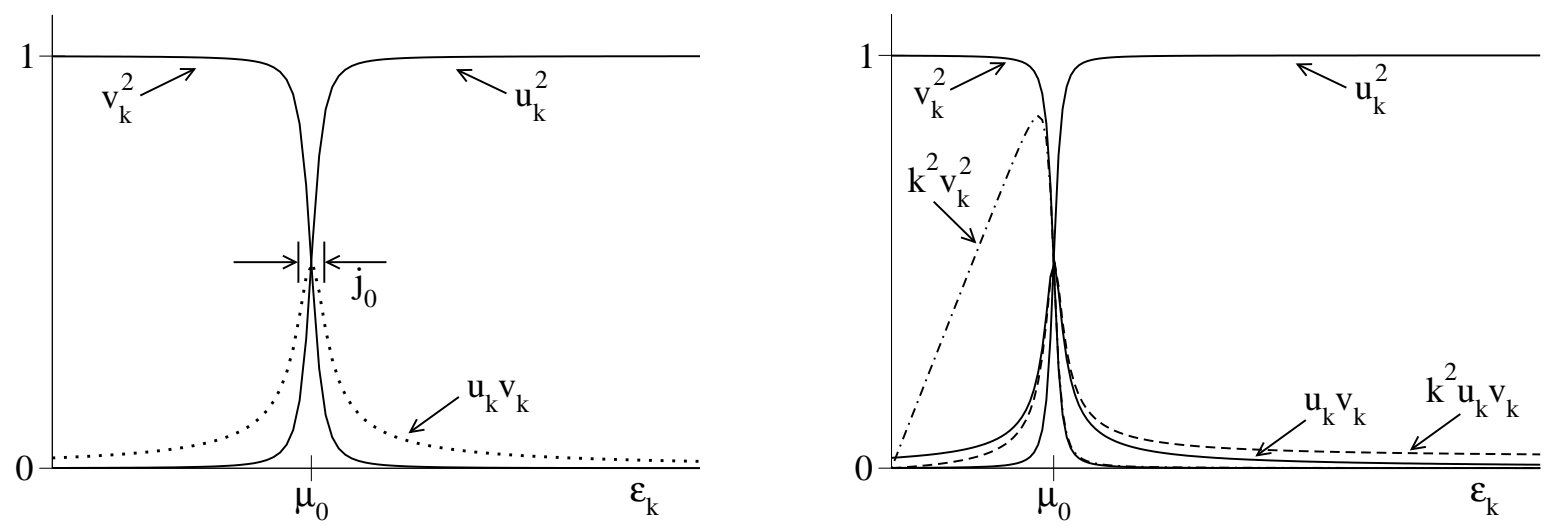

FIG. 9: Left: Plot of $u_{k}^{2}$ and $v_{k}^{2}$ along with $u_{k} v_{k}$. Note: the width labeled " $j_{0}$ " is exaggerated to make it more visible. Right: Same plot with $k^{2} v_{k}^{2}$ (dotdashes) and $k^{2} u_{k} v_{k}$ added, with an extended $x$-axis.

with $|\mathbf{k}|=\left|\mathbf{k}^{\prime}\right|=k_{\mathrm{F}}$ in the inner integral. This means that $E_{\mathbf{k}}, E_{\mathbf{k}^{\prime}} \rightarrow 0$ and the $u^{2}$ and $v^{2}$ terms become theta functions. As suggested by Fig. 10, the inner integral has the form of a non-interacting density-density correlator or Lindhard function. Such a correlator is defined and evaluated in Ref. [45] for non-interacting $T=0$ Green's functions $G_{0}$ :

$$
\begin{aligned}
& \Pi^{0}\left(\mathbf{q}, q_{0}\right)=-2 i \int \frac{d^{4} k}{(2 \pi)^{4}} G^{0}(k) G^{0}(k+q) \\
&=2 \int \frac{d^{3} k}{(2 \pi)^{3}} \theta\left(|\mathbf{q}+\mathbf{k}|-k_{\mathrm{F}}\right) \theta\left(k_{\mathrm{F}}-|\mathbf{k}|\right) \\
& \times\left(\frac{1}{q_{0}+\varepsilon_{\mathbf{k}}-\varepsilon_{\mathbf{q}+\mathbf{k}}+i \eta}-\frac{1}{q_{0}+\varepsilon_{\mathbf{q}+\mathbf{k}}-\varepsilon_{\mathbf{k}}-i \eta}\right) .
\end{aligned}
$$

Because of the peaking at $\mu_{0}$, the leading contribution is

$$
\begin{aligned}
\frac{1}{\beta V}\left[\Gamma_{2}\right]_{\text {induced }} & =-\left.\frac{1}{2}\left(C_{0}^{(1)}\right)^{2} \int \frac{d^{3} k}{(2 \pi)^{3}} u_{k} v_{k} \int \frac{d^{3} k^{\prime}}{(2 \pi)^{3}} u_{k^{\prime}} v_{k^{\prime}} \Pi^{0}\left(\mathbf{k}-\mathbf{k}^{\prime}, 0\right)\right|_{|\mathbf{k}|=\left|\mathbf{k}^{\prime}\right|=k_{\mathrm{F}}} \\
& =-\frac{1}{8}\left(C_{0}^{(1)}\right)^{2} \phi_{\mathrm{B}}^{2}\left\langle\Pi_{0}\left(\mathbf{k}-\mathbf{k}^{\prime}\right)\right\rangle_{|\mathbf{k}|=\left|\mathbf{k}^{\prime}\right|=k_{\mathrm{F}}},
\end{aligned}
$$

where $\langle\cdots\rangle$ denotes an average over the angle between $\mathbf{k}$ and $\mathbf{k}^{\prime}$. Using the expressions in Ref. [45] and defining $\eta \equiv \sqrt{\left(k_{\mathrm{F}}^{2}-\mathbf{k} \cdot \mathbf{k}^{\prime}\right) / 2 k_{\mathrm{F}}^{2}}$,

$$
\begin{aligned}
\left\langle\Pi_{0}\left(\mathbf{k}-\mathbf{k}^{\prime}\right)\right\rangle_{|\mathbf{k}|=\left|\mathbf{k}^{\prime}\right|=k_{\mathrm{F}}} & =\frac{M k_{\mathrm{F}}}{2 \pi^{2}} \int_{0}^{1} d \eta\left[\eta+\frac{1-\eta^{2}}{2} \ln \left(\frac{1+\eta}{1-\eta}\right)\right] \\
& =\frac{M k_{\mathrm{F}}}{2 \pi^{2}} \frac{1}{3}(1+2 \ln 2) .
\end{aligned}
$$

From Eq. (155), the leading order (LO) result for $\left|j_{0} / \mu_{0}\right|$ at $T=0$ is

$$
\left|j_{0} / \mu_{0}\right|_{L O}=\frac{8}{e^{2}} e^{-\pi / 2 k_{\mathrm{F}}\left|a_{s}\right|}
$$




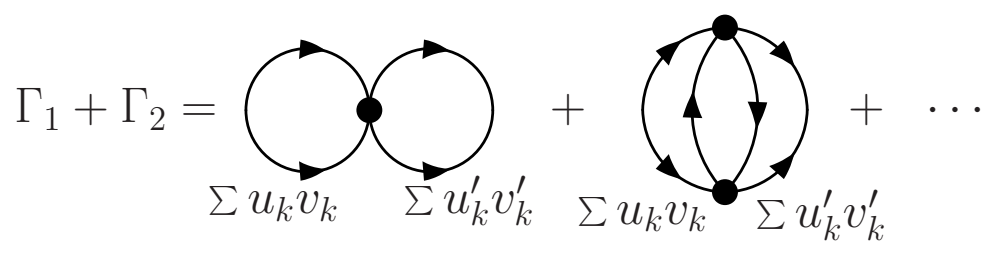

FIG. 10: Contributions to $\Gamma_{1}$ and $\Gamma_{2}$ with logarithmic divergences at the Fermi surface as $j_{0} \rightarrow 0$. The vertices in each case have coefficient $C_{0}^{(1)}$.

After adding the renormalizations to $\Gamma_{2}$ that take $\phi_{\mathrm{B}}$ to $\phi$, we find that Eq. (52) becomes at NLO

$$
j_{0}=-\left(j_{1}+j_{2}\right)=-\frac{1}{2}\left|C_{0}^{(1)}\right| \phi\left[1-\left|C_{0}^{(1)}\right| \frac{M k_{\mathrm{F}}}{2 \pi^{2}}\left(\frac{1+2 \ln 2}{3}\right)\right] .
$$

The final result for $\left|j_{0} / \mu_{0}\right|$ is that the exponent is modified, which changes the prefactor, yielding

$$
\left|j_{0} / \mu_{0}\right|_{N L O} \approx \frac{1}{(4 e)^{1 / 3}}\left|j_{0} / \mu_{0}\right|_{L O}
$$

or, associating $j_{0}$ with the gap again (see below about the gap in a Kohn-Sham DFT),

$$
\Delta_{N L O} \approx \frac{1}{(4 e)^{1 / 3}} \Delta_{L O}
$$

Thus, we recover the universal suppression of the gap in the $k_{\mathrm{F}} \rightarrow 0$ limit found in Refs. [41, 42].

\section{SUMMARY}

In this paper, we extend the effective field theory treatment of a uniform, dilute Fermi gas to include pairing. The starting point is a generating functional for the grand canonical partition function including a chemical potential for the fermion number and a source coupled to the pair density, which we take to be constant. Legendre transformations with respect to the sources lead to an effective action of the fermion and pair densities.

The effective action formalism is often used in condensed matter applications to discuss superconductivity. In that case, the pairing field enters as an auxiliary field. After integrating out the fermion fields, a conventional one-particle-irreducible effective action is derived by Legendre transformation with respect to the auxiliary field. A minimum at nonzero expectation value of this field (which is proportional to the pairing gap) indicates spontaneously symmetry breaking of the phase symmetry related to fermion number conservation, which implies the normal ground state is unstable to pairing.

In contrast, the calculation here is carried out by adapting the inversion method proposed in Ref. [19] to an EFT treatment of the dilute Fermi gas. The inversion method in Ref. [19] was applied to conventional BCS superconductivity using a source coupled to the pair creation and destruction operator. Instead of organizing the perturbative inversion in terms of a coupling constant (e.g., the electron charge squared), we use the EFT expansion parameter for the natural dilute system, which is the inverse of the resolution scale. In the uniform system, the ultimate dimensionless expansion parameters are products of the Fermi 
momentum $k_{\mathrm{F}}$ and parameters of the effective range expansion (e.g., the scattering length $a_{s}$, which is the order of the interaction range for a natural system).

We encounter a new renormalization problem because of ultraviolet divergences involving the composite pair density. To deal with it, a new term proportional to the square of the external source $j$, with new coupling constant $\zeta$, is added to the Lagrangian. In our renormalization prescription, we implicitly assume that $\zeta$ has a Taylor expansion in $a_{s}$ to be consistent with the inversion method. On dimensional grounds, a term with $\zeta$ proportional to $1 / a_{s}$ is possible, which would be independent of $\Lambda$. Such a term is consistent with the development for effective actions with local composite operators in Refs. [33, 34], where the coefficient of analogous terms have a Laurent expansion starting at the inverse of the coupling. However, the counterterms in our prescription along with the source-pair-density counterterms suffice for us to renormalize, with the prescription that the pair density is unchanged from the non-interacting system. We verify the renormalization through NLO by using the PDS subtraction prescription with dimensional regularization.

At leading order, we reproduce the results of Papenbrock and Bertsch [40] for the gap and energy density. At next-to-leading order, we reproduce the leading weak-coupling limit derived by Gor'kov and Melik-Barkhudarov [41] for the modification of the gap, and generate complete expressions for the observables suitable for numerical evaluation and application to finite systems in the local density approximation (LDA). We have focused on the zero temperature limit here, but the formalism applies directly at finite temperature. For example, we can find the transition temperature by looking for where the non-trivial $\left(j_{0} \neq 0\right)$ solution to the $j_{0}$ self-consistency equation disappears, which corresponds to solving for the temperature with $j_{0}$ set to zero.

The next step is to extend our results to finite systems. This means merging the formalism here with the density functional formalism of Refs. [9, 10, 11]. The renormalization will carry over from the present discussion via the LDA, as in Ref. [9]. In practice, efficient subtraction schemes at leading order have been developed by Bulgac and $\mathrm{Yu}$ [55, 56, 57].

The extension to higher-order terms, such as $C_{2}$ and $C_{2}^{\prime}$ requires further renormalization. In general, we expect that every term of equal or lower dimension, consistent with symmetries, to enter. When $C_{2}$ is added, for example, in the general case a new source term proportional to $C_{2} j^{2} \psi^{\dagger} \psi$ is needed. Extending to isospin and spin-dependent interactions is straightforward formally, but the renormalization issues should be reconsidered at each stage.

We have noted that the Kohn-Sham potential $j_{0}$ plays the same role at leading order that a gap does in a BCS treatment with a contact interaction. The conventional definition of a gap function is an integral over the potential and the anomalous Green's function. For example, Mahan defines for a local potential $V(\mathbf{q})[54]$ :

$$
\Delta(\mathbf{q})=-\frac{1}{V} \sum_{\mathbf{k}} V(\mathbf{k}) \mathcal{F}_{0}(\mathbf{q}-\mathbf{k}, \tau=0)=-\int \frac{d^{4} k}{(2 \pi)^{4}} V(\mathbf{q}-\mathbf{k}) \mathcal{F}_{0}\left(k_{0}, \mathbf{k}\right) e^{i k_{0} \eta}
$$

Given a momentum expansion of $V$, we could define a corresponding series of gap functions (see the appendix of Ref. [40]). However, it is important to note that the Kohn-Sham "gaps" are not directly connected to the single-particle spectrum. In Ref. [11], the connection between the Kohn-Sham Green's function and the full single-particle Green's function is discussed. There is no difference at LO, but at NLO and higher orders the self-energies will differ, which means there is no longer a direct correspondence between the Kohn-Sham gap function and that extracted from the full Green's function. The systematics of the differences 
between these gaps (and also a gap defined from even-odd staggering of ground-state energies in finite systems) have yet to be investigated.

The present formalism is not the only path to a nuclear DFT including pairing. As discussed in the introduction, an alternative to a local source coupled to the local pair density is to use a nonlocal source and density [28, 29]. Proceeding in this way would parallel the response in particle theory to problems uncovered by Banks and Raby, which was to focus on CJT two-particle-irreducible effective actions. An alternative would be to apply the auxiliary field approach in the pairing channel. Here a clean separation of particlehole and particle-particle channels may be the problem, although this is regularly a part of phenomenological approaches. Finally, a completely different path to DFT is outlined by Polonyi and Schwenk, who propose a renormalization group method [58]. These avenues are all worth exploring.

\section{Acknowledgments}

We thank S. Bogner, A. Bulgac, A. Schwenk, and B. Serot for useful comments and discussions. This work was supported in part by the National Science Foundation under Grants No. PHY-0098645 and No. PHY-0354916, by the DFG through funds provided to the SFB/TR 16 "Subnuclear structure of matter", and by the BMBF under contract number $06 \mathrm{BN} 411$.

\section{APPENDIX A: CANONICAL DERIVATION OF $W_{0}\left[\mu_{0}, j_{0}\right]$}

In this appendix, we derive an expression for $W_{0}\left[\mu_{0}, j_{0}\right] / \beta$, which is proportional to the logarithm of the partition function of the noninteracting system in the presence of $\mu_{0}$ and $j_{0}$, in the zero temperature $(\beta \rightarrow 0)$ limit. If $\mu_{0} \neq 0$ but $j_{0}=0$, we project onto the normal noninteracting ground state, which is simple to construct. In the uniform case, we just fill the lowest plane-wave states (which have the lowest eigenvalues) up to the Fermi momentum $k_{\mathrm{F}}$, which is related to the density by Eq. (11). This minimizes the energy for a given particle number. When $j_{0} \neq 0$, we need to construct the state that minimizes the free energy for given chemical potential $\mu_{0}$ (and given $j_{0}$ ). Here we follow the notation and general discussion of Ref. [45] in using the Bogoliubov transformation, which finds the exact diagonalization in the noninteracting case with constant $j_{0}$. Not surprisingly, the state that

minimizes $\widehat{K}_{0} \equiv \widehat{H}_{0}-\mu_{0} \widehat{N}$, where $\widehat{H}_{0}$ includes the $j_{0}$ dependence, is a BCS-type variational state.

The non-interacting $\widehat{K}_{0}$ in terms of creation and destruction operators for the momentum states with spin up and spin down is:

$$
\widehat{K}_{0}=\sum_{\mathbf{k} \alpha}\left(\varepsilon_{\mathbf{k}}^{0}-\mu_{0}\right) a_{\mathbf{k} \alpha}^{\dagger} a_{\mathbf{k} \alpha}+\sum_{\mathbf{k}} j_{0}\left(a_{\mathbf{k} \uparrow}^{\dagger} a_{-\mathbf{k} \downarrow}^{\dagger}+a_{-\mathbf{k} \downarrow} a_{\mathbf{k} \uparrow}\right)+\frac{1}{2} \zeta^{(0)} j_{0}^{2} V,
$$

with $\varepsilon_{\mathbf{k}}^{0}=k^{2} / 2 M$. (This definition generalizes $\widehat{K}_{0} \equiv \widehat{H}_{0}-\mu_{0} \widehat{N}$ from Ref. [45].) This result is apparent from inspection, but can be derived formally from the field expansions for $\psi(\mathbf{x})$ and $\psi^{\dagger}(\mathbf{x})$. The term proportional to $j_{0}^{2}$ is needed to ensure $\Lambda$-independent results, but plays no explicit role here. The minimized expectation value of $\widehat{K}_{0}$ at fixed $V$ and $\mu$ at $T=0$ is 
related to the energy by

$$
\frac{1}{\beta} W_{0}\left[\mu_{0}, j_{0}\right] \stackrel{\beta \rightarrow \infty}{\longrightarrow}\left\langle\widehat{K}_{0}\right\rangle=\Omega_{0}(\mu, T=0, V)=\left.\left(E_{0}-\mu_{0} N\right)\right|_{T=0} .
$$

We introduce the Bogoliubov-transformed operators $\alpha_{\mathbf{k}}$ and $\beta_{\mathbf{k}}$,

$$
\alpha_{\mathbf{k}}=u_{k} a_{\mathbf{k} \uparrow}-v_{k} a_{-\mathbf{k} \downarrow}^{\dagger}, \quad \quad \beta_{\mathbf{k}}=u_{k} a_{-\mathbf{k} \downarrow}+v_{k} a_{\mathbf{k} \uparrow}^{\dagger},
$$

so that

$$
a_{\mathbf{k} \uparrow}=u_{k} \alpha_{\mathbf{k}}+v_{k} \beta_{-\mathbf{k}}^{\dagger}, \quad \quad a_{-\mathbf{k} \downarrow}=u_{k} \beta_{-\mathbf{k}}-v_{k} \alpha_{\mathbf{k}}^{\dagger} .
$$

The anticommutation relations

$$
\left\{\alpha_{\mathbf{k}}, \alpha_{\mathbf{k}^{\prime}}^{\dagger}\right\}=\left\{\beta_{\mathbf{k}}, \beta_{\mathbf{k}^{\prime}}^{\dagger}\right\}=\delta_{\mathbf{k k}^{\prime}}
$$

with all others equal to zero, imply that

$$
u_{k}^{2}+v_{k}^{2}=1 .
$$

The BCS ground state is defined so that

$$
\alpha_{\mathbf{k}}|\mathrm{BCS}\rangle=\beta_{\mathbf{k}}|\mathrm{BCS}\rangle=0 \text {. }
$$

(See Ref. 45] for an explicit construction in terms of the $a^{\prime}$ 's and $a^{\dagger}$ 's.) At this point we don't know that this is actually the ground state of the noninteracting system with non-zero $j_{0}$, but we do not need to make this assumption.

By following the normal-ordering approach in Ref. [45] or simply by applying the commutation relations, we can rewrite $\widehat{K}_{0}$ with no loss of generality as:

$$
\widehat{K}_{0}=U+\widehat{H}_{1}+\widehat{H}_{2}
$$

where

$$
\begin{aligned}
U & =2 \sum_{\mathbf{k}} \xi_{k} v_{k}^{2}+2 j_{0} \sum_{\mathbf{k}} u_{k} v_{k}+\frac{1}{2} \zeta^{(0)} j_{0}^{2} V \\
\widehat{H}_{1} & =\sum_{\mathbf{k}}\left(\alpha_{\mathbf{k}}^{\dagger} \alpha_{\mathbf{k}}+\beta_{-\mathbf{k}}^{\dagger} \beta_{-\mathbf{k}}\right)\left[\left(u_{k}^{2}-v_{k}^{2}\right) \xi_{k}-2 u_{k} v_{k} j_{0}\right] \\
\widehat{H}_{2} & =\sum_{\mathbf{k}}\left(\alpha_{\mathbf{k}}^{\dagger} \beta_{-\mathbf{k}}^{\dagger}+\beta_{-\mathbf{k}} \alpha_{\mathbf{k}}\right)\left[2 u_{k} v_{k} \xi_{k}+j_{0}\left(u_{k}^{2}-v_{k}^{2}\right)\right]
\end{aligned}
$$

In these expressions,

$$
\xi_{k}=\varepsilon_{\mathbf{k}}^{0}-\mu_{0} .
$$

We still have freedom in our choice of $u_{k}$ and $v_{k}$, subject to the constraint (A6). Using this freedom, we can choose

$$
2 u_{k} v_{k} \xi_{k}=-\left(u_{k}^{2}-v_{k}^{2}\right) j_{0}
$$

so that $\widehat{H}_{2}$ vanishes identically. This condition is satisfied for

$$
u_{k} v_{k}=-\frac{j_{0}}{2 E_{k}}, \quad u_{k}^{2}=\frac{1}{2}\left(1+\frac{\xi_{k}}{E_{k}}\right), \quad v_{k}^{2}=\frac{1}{2}\left(1-\frac{\xi_{k}}{E_{k}}\right)
$$


with

$$
E_{k} \equiv \sqrt{\xi_{k}^{2}+j_{0}^{2}}
$$

Substituting from (A14) into Eq. (A10), we find that $\widehat{H}_{1}$ simplifies to

$$
\widehat{H}_{1}=\sum_{\mathbf{k}} E_{k}\left(\alpha_{\mathbf{k}}^{\dagger} \alpha_{\mathbf{k}}+\beta_{-\mathbf{k}}^{\dagger} \beta_{-\mathbf{k}}\right)
$$

Then it is clear from this equation together with Eq. (A7) that the corresponding $|\mathrm{BCS}\rangle$ state (which is dependent on $\mu_{0}$ and $j_{0}$ ) will be the ground state of $\widehat{K}_{0}\left(\mu_{0}, j_{0}\right)$, since $U$ is a $c$-number, $E_{k}$ is positive definite, and $\left\langle\widehat{H}_{2}\right\rangle \equiv 0$.

Note that there is no self-consistent gap equation at this point; for any given value of $\mu_{0}$ and $j_{0}$, we can construct this ground state with Eq. (A14) satisfied. The density of fermions follows immediately as

$$
\rho=\frac{N}{V}=\frac{1}{V} \sum_{\mathbf{k} \alpha}\left\langle\mathrm{BCS}\left|a_{\mathbf{k} \alpha}^{\dagger} a_{\mathbf{k} \alpha}\right| \mathrm{BCS}\right\rangle=\frac{2}{V} \sum_{\mathbf{k}} v_{k}^{2}=\frac{1}{V} \sum_{k}\left(1-\frac{\xi_{k}}{E_{k}}\right) .
$$

and the ground-state expectation value of $\widehat{K}_{0}$, which is equal to $U$, is

$$
\begin{aligned}
U & =\left\langle\mathrm{BCS}\left|\widehat{K}_{0}\right| \mathrm{BCS}\right\rangle=\left.\left(\frac{1}{\beta} W_{0}\left[\mu_{0}, j_{0}\right]\right)\right|_{\beta \rightarrow \infty} \\
& =2 \sum_{\mathbf{k}} \xi_{k} v_{k}^{2}+2 j_{0} \sum_{\mathbf{k}} u_{k} v_{k}+\frac{1}{2} \zeta^{(0)} j_{0}^{2} V \\
& =\sum_{\mathbf{k}}\left(\xi_{k}-E_{k}\right)+\frac{1}{2} \zeta^{(0)} j_{0}^{2} V .
\end{aligned}
$$

Upon converting the sums to integrals and dividing by the volume, we obtain the expression for $W_{0} / \beta V$ in Eq. (33). We emphasize that there is no approximation or truncation here.

\section{APPENDIX B: INTEGRALS FOR DR/PDS}

Here we generalize the DR/MS integrals used in Ref. [40] to the PDS subtraction scheme. The starting point is the integral [40]

$$
\int_{0}^{\infty} \frac{\varepsilon^{\alpha} d \varepsilon}{\sqrt{(\varepsilon-a)^{2}+b^{2}}}=\left(a^{2}+b^{2}\right)^{\alpha / 2}\left(\frac{-\pi}{\sin \pi \alpha}\right) P_{\alpha}^{0}\left(-a / \sqrt{a^{2}+b^{2}}\right),
$$

where $P_{\alpha}^{0}$ is a Legendre function. The right side of Eq. (B1) can be analytically continued in $\alpha$ to define the relevant integrals in $D$ dimensions. For appropriate $D$, there is an analytic formula, and then the answer is continued back to the relevant number of spatial dimensions after the approprite subtraction of poles (according to the prescription).

A particular integral of interest with $\alpha=0$ is

$$
\left(\frac{\Lambda}{2}\right)^{3-D} \int \frac{d^{D} k}{(2 \pi)^{3}} \frac{1}{\sqrt{\left(\varepsilon_{\mathbf{k}}-\mu_{0}\right)^{2}+j_{0}^{2}}}=\left(\frac{2 M}{4 \pi}\right)^{D / 2} \frac{(\Lambda / 2)^{3-D}}{\Gamma(D / 2)} \int_{0}^{\infty} \frac{\varepsilon^{(D / 2-1)} d \varepsilon}{\sqrt{\left(\varepsilon-\mu_{0}\right)^{2}+j_{0}^{2}}}
$$




$$
\begin{gathered}
=\left(\frac{2 M \mu_{0}}{4 \pi}\right)^{D / 2} \frac{(\Lambda / 2)^{3-D}}{\Gamma(D / 2)}\left(\frac{-\pi}{\sin \pi(D / 2-1)}\right) \\
\times \frac{\left(1+x^{2}\right)^{(D / 2-1) / 2}}{\mu_{0}} P_{D / 2-1}^{0}\left(\frac{-1}{\sqrt{1+x^{2}}}\right),
\end{gathered}
$$

where $x \equiv\left|j_{0} / \mu_{0}\right|$. To apply Eq. (B2) in the PDS scheme, we must subtract poles in $(2-D)$. The only such pole comes from

$$
\frac{-\pi}{\sin \pi(D / 2-1)} \longrightarrow \frac{2}{2-D}+\frac{\pi^{2}}{12}(2-D)+\mathcal{O}\left[(2-D)^{2}\right]
$$

which means the pole term is (all other factors evaluated at $D=2$ ):

$$
\left(\frac{-\Lambda}{2}\right) \frac{1}{\mu_{0}}\left(\frac{-2}{2-D}\right) \frac{2 M \mu_{0}}{4 \pi}=-\frac{M \Lambda}{2 \pi} \frac{1}{(D-2)} \stackrel{D \rightarrow 3}{\longrightarrow}-\frac{M \Lambda}{2 \pi} .
$$

More generally, the basic DR/PDS integral in $D$ dimensions, with $x \equiv j_{0} / \mu_{0}$, is

$$
\begin{aligned}
I(\beta) \equiv\left(\frac{\Lambda}{2}\right)^{3-D} \int \frac{d^{D} k}{(2 \pi)^{D}} \frac{\left(\varepsilon_{\mathbf{k}}^{0}\right)^{\beta}}{E_{k}}=\frac{M \Lambda}{2 \pi}\left(\mu_{0}\right)^{\beta}\left(1-\delta_{\beta, 2} \frac{x^{2}}{2}\right) \\
+(-)^{\beta+1} \frac{M^{3 / 2}}{\sqrt{2} \pi}\left[\mu_{0}^{2}\left(1+x^{2}\right)\right]^{(\beta+1 / 2) / 2} P_{\beta+1 / 2}^{0}\left(\frac{-1}{\sqrt{1+x^{2}}}\right) \\
\equiv \frac{M \Lambda}{2 \pi}\left(\mu_{0}\right)^{\beta}\left(1-\delta_{\beta, 2} \frac{x^{2}}{2}\right)+\widetilde{I}(\beta),
\end{aligned}
$$

where $\widetilde{I}(\beta)$ is the $\Lambda$-independent part of $I(\beta)$

We can expand $\widetilde{I}(\beta)$ with $\beta=0,1,2$ for small $x$ as:

$$
\begin{aligned}
& \widetilde{I}(0)=-\frac{\left(2 M \mu_{0}\right)^{1 / 2} M}{\pi^{2}}\left[\left(2+\log \frac{x}{8}\right)+\frac{1}{16}\left(1+\log \frac{x}{8}\right) x^{2}+\cdots\right], \\
& \widetilde{I}(1)=-\frac{\left(2 M \mu_{0}\right)^{3 / 2}}{2 \pi^{2}}\left[\left(\frac{8}{3}+\log \frac{x}{8}\right)+\frac{3}{16}\left(1-\log \frac{x}{8}\right) x^{2}+\cdots\right], \\
& \widetilde{I}(2)=-\frac{\left(2 M \mu_{0}\right)^{3 / 2} \mu_{0}}{2 \pi^{2}}\left[\left(\frac{46}{15}+\log \frac{x}{8}\right)-\frac{15}{16}\left(1+\log \frac{x}{8}\right) x^{2}+\cdots\right] .
\end{aligned}
$$

[1] G. P. Lepage, "What is Renormalization?", in From Actions to Answers (TASI-89), edited by T. DeGrand and D. Toussaint (World Scientific, Singapore, 1989); "How to Renormalize the Schrödinger Equation", [nucl-th/9706029].

[2] S. R. Beane, P. F. Bedaque, W. C. Haxton, D. R. Phillips, and M. J. Savage, "From Hadrons to Nuclei: Crossing the Border", [nucl-th/0008064].

[3] P. F. Bedaque and U. van Kolck, Ann. Rev. Nucl. Part. Sci. 52 (2002) 339 [nucl-th/0203055]. 
[4] D. B. Kaplan, "Five lectures on effective field theory," nucl-th/0510023.

[5] E. Epelbaum, Prog. Part. Nucl. Phys. 57 (2006) 654 [nucl-th/0509032].

[6] E. Braaten and H.-W. Hammer, Phys. Rept. 428 (2006) 259 [cond-mat/0410417].

[7] H.-W. Hammer and R.J. Furnstahl, Nucl. Phys. A 678 (2000) 277 [nucl-th/0004043].

[8] R.J. Furnstahl and H.-W. Hammer, Ann. Phys. (NY) 302 (2002) 206 [nucl-th/0208058] .

[9] S. Puglia, A. Bhattacharyya, and R.J. Furnstahl, Nucl. Phys. A 723 (2003) 145 [nucl-th/0212071].

[10] A. Bhattacharyya and R.J. Furnstahl, Nucl. Phys. A 747 (2005) 268 [nucl-th/0408014].

[11] A. Bhattacharyya and R.J. Furnstahl, Phys. Lett. B 607 (2005) 259 [nucl-th/0410105].

[12] S. Coleman, Aspects of Symmetry (Cambridge Univ. Press, 1988).

[13] M.E. Peskin and D.V. Schroeder, An Introduction to Quantum Field Theory (Addison-Wesley, 1995).

[14] S. Weinberg, The Quantum Theory of Fields: vol. II, Modern Applications (Cambridge University Press, 1996).

[15] N. Nagaosa, Quantum Field Theory in Condensed Matter Physics (Springer Verlag, 1999).

[16] M. Stone, The Physics of Quantum Fields (Springer Verlag, 2000)

[17] R. Fukuda, T. Kotani, Y. Suzuki, and S. Yokojima, Prog. Theor. Phys. 92 (1994) 833.

[18] R. Fukuda, M. Komachiya, S. Yokojima, Y. Suzuki, K. Okumura, and T. Inagaki, Prog. Theor. Phys. Suppl. 121 (1995) 1.

[19] T. Inagaki and R. Fukuda, Phys. Rev. B 46 (1992) 10931.

[20] M. Valiev and G. W. Fernando, cond-mat/9702247.

[21] M. Valiev and G. W. Fernando, Phys. Lett. A 227 (1997) 265.

[22] M. Valiev and G. W. Fernando, Phys. Rev. B 54 (1996) 7765.

[23] R. Chitra and G. Kotliar, Phys. Rev. B 62 (2000) 12715.

[24] R. Chitra and G. Kotliar, Phys. Rev. B 63 (2001) 115110.

[25] J. Polonyi and K. Sailer, cond-mat/0108179.

[26] W. Kohn and J. M. Luttinger, Phys. Rev. 118 (1960) 41.

[27] J. M. Luttinger and J. C. Ward, Phys. Rev. 118 (1960) 1417.

[28] L. N. Oliveira, E. K. U. Gross, and W. Kohn, Phys. Rev. Lett. 60 (1988) 2430.

[29] S. Kurth, M. Marques, M. Lüders, and E. K. U. Gross, Phys. Rev. Lett. 83 (1999) 2628.

[30] M. V. Stoitsov, J. Dobaczewski, W. Nazarewicz, S. Pittel and D. J. Dean, Phys. Rev. C 68 (2003) 054312, and references therein.

[31] J. C. Collins, Renormalization (Cambridge Univ. Press, 1986).

[32] T. Banks and S. Raby, Phys. Rev. D 14 (1976) 2182.

[33] H. Verschelde, Phys. Lett. B 351 (1995) 242.

[34] H. Verschelde, S. Schelstraete, and M. Vanderkelen, Z. Phys. C 76 (1997) 161.

[35] K. Knecht and H. Verschelde, Phys. Rev. D 64 (2001) 085006.

[36] V. A. Miransky, Int. J. Mod. Phys. A 8 (1993) 135.

[37] V. A. Miransky and K. Yamawaki, Phys. Rev. D 55 (1997) 5051 [Erratum-ibid. D 56 (1997) 3768].

[38] V. P. Gusynin, V. A. Miransky and A. V. Shpagin, Phys. Rev. D 58 (1998) 085023.

[39] M. Marini, F. Pistolesi, and G. C. Strinati, Eur. Phys. J. B 1 (1998) 151.

[40] T. Papenbrock and G.F. Bertsch, Phys. Rev. C 59 (1999) 2052.

[41] L. P. Gor'kov and T. K. Melik-Barkhudarov, Sov. Phys. JETP 13 (1961) 1018.

[42] H. Heiselberg, C. J. Pethick, H. Smith, and L. Viverit, Phys. Rev. Lett. 85 (2000) 2418.

[43] R.J. Furnstahl, H.-W. Hammer and N. Tirfessa, Nucl. Phys. A 689 (2001) 846 
[nucl-th/0010078].

[44] J. W. Negele and H. Orland, Quantum Many-Particle Systems (Addison-Wesley, New York, 1988).

[45] A. L. Fetter and J. D. Walecka, Quantum Theory of Many-Particle Systems (McGraw-Hill, New York, 1971).

[46] K. Okumura, Int. J. Mod. Phys. A 11 (1996) 65.

[47] S. Yokojima, Phys. Rev. D 51 (1995) 2996.

[48] M. Bender, P. H. Heenen, and P.-G. Reinhard, Rev. Mod. Phys. 75 (2003) 121.

[49] D. B. Kaplan, M. J. Savage and M. B. Wise, Phys. Lett. B 424 (1998) 390 [nucl-th/9801034]; Nucl. Phys. B 534 (1998) 329 [nucl-th/9802075].

[50] U. van Kolck, Nucl. Phys. A 645 (1999) 273 [nucl-th/9808007].

[51] J. Gegelia, Phys. Lett. B 429 (1998) 227; J. Phys. G 25 (1999) 1681 [nucl-th/9805008].

[52] E. Braaten, private communication.

[53] J. Zinn-Justin, Quantum Field Theory and Critical Phenomena 4th ed. (Oxford University Press, New York, 2002), ch. 12.

[54] G. D. Mahan, Many-Particle Physics (Plenum, New York, 2000).

[55] Y. Yu and A. Bulgac, Phys. Rev. Lett. 90 (2003) 222501 [nucl-th/0210047].

[56] A. Bulgac, Phys. Rev. C 65 (2002) 051305 [nucl-th/0108014].

[57] A. Bulgac and Y. Yu, Phys. Rev. Lett. 88 (2002) 042504 [nucl-th/0106062].

[58] A. Schwenk and J. Polonyi, nucl-th/0403011. 